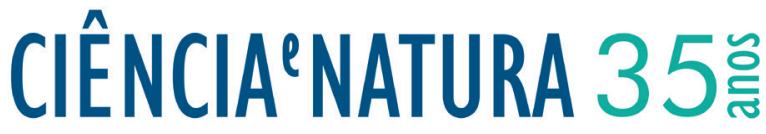

\section{Solução para as Equações de Navier-Stokes em domínios tridimensionais finos}

\author{
Solutions for the Navier-Stokes Equations in tridimensional thin domains
}

\author{
Felipe Crivellaro Minuzzi*1, João Paulo Lukaszczyk ${ }^{2}$, \\ ${ }^{1}$ Acadêmico do Mestrado em Matemática, Universidade Federal de Santa Maria, Santa Maria, Brasil. \\ Professor Associado do Departamento de Matemática, Universidade Federal de Santa Maria, Santa Maria, Brasil.
}

\begin{abstract}
Resumo
A forma clássica do sistema de equações de Navier-Stokes, o qual deriva do princípio de conservação de massa e momento linear, descreve o movimento de um fluido homogêneo sujeito a um campo de forças externas. Neste trabalho, desenvolve-se um estudo para encontrar o intervalo maximal de existência de soluções no tempo para as equações de Navier-Stokes em um domínio tridimensional fino, isto é, $\Omega_{\epsilon}=\omega \times(0, \epsilon)$, onde $\omega \subset \mathbb{R}^{2} e \epsilon \in(0,1)$, considerando combinações de diferentes condições de fronteira.
\end{abstract}

Palavras-chave: Equações de Navier-Stokes, Domínios finos, Existência de solução.

\begin{abstract}
The classical form of the Navier-Stokes equations system, which is derived from the principle of conservation of mass and momentum, describes the motion of a homogeneous fluid subject to a field of external forces. In this work, we develop a study to find the maximal interval of existence of solutions in time to the Navier-Stokes equations in a three dimensional thin domain, i.e., $\Omega_{\epsilon}=\omega \times(0, \epsilon)$, where $\omega \subset \mathbb{R}^{2} e \epsilon \in(0,1)$, considering different combinations of boundary conditions.
\end{abstract}

Keywords: Navier-Stokes equations; Thin domains; Existence of solutions. 


\section{Introdução}

$\mathrm{P}$ or volta dos séculos XVIII e XIX, os matemáticos Leonhard Paul Euler (1707 - 1783), Claude Louis Marie Henri Navier (1785 - 1836) e George Gabriel Stokes (1819 - 1903) fizeram grandes avanços no campo da mecânica dos fluidos. O primeiro foi responsável pela dedução do movimento de fluidos quando estes não são viscosos, enquanto os outros dois consideraram o efeito da viscosidade. O resultado de seus trabalhos são o que hoje chamamos de Equações de Euler e Equações de Navier-Stokes, esta, objeto de estudo deste trabalho.

As equações de Navier-Stokes descrevem o movimento de um fluido homogêneo, sujeito a um campo de forças externos, e são deduzidas a partir da $2^{\mathrm{a}}$ Lei de Newton, do Princípio de Conservação da Massa e de Momento Linear, como pode ser visto em Melo e Neto (1991), Chorin e Marsden (1990), Fox e McDonald (1988) e Kreiss e Lorenz (1989). Sua importância não atinge apenas a Matemática, mas campos como Física, Dinâmica dos Fluidos, Meteorologia, e, mais específicos, Dinâmica dos Oceanos, Geofísica, Mecânica dos Sólidos e Fisiologia, entre outros.

Apesar de muito usada, quando falamos em existência de soluções clássicas em $\mathbb{R}^{3} \times[0, \infty)$, este ainda é um problema em aberto da Matemática, conhecido por ser um dos Millenium Problems do Clay Mathematical Institute, que premia 1 milhão de dólares a quem resolvê-lo. $\mathrm{O}$ trabalho da matemática russa Olga Ladyzhenskaya foi o primeiro que provou de maneira rigorosa a convergência de um método de diferenças finitas para as equações de Navier-Sokes. Hoje em dia, são conhecidos resultados de existência e unicidade para solução fraca em espaços de Sobolev, como pode ser encontrado em Lions (1969) e Temam (1984), os quais usam o método de Galerkin. Recentemente, em janeiro de 2014, o matemático cazáque Muchtarbai Otelbajew publicou um artigo afirmando ter encontrado solução clássica para as equações de NavierStokes. Tal trabalho ainda está em análise pela comissão examinadora do Clay Mathematical Institute.

A forma vetorial das equações de Navier-Stokes é dadas por

$\begin{cases}u_{t}+u \cdot \nabla u-\mu \Delta u+\nabla p=f, & \text { em } \Omega \times(0, \infty) \\ \operatorname{div} u=0, & \text { em } \Omega \times(0, \infty)\end{cases}$

onde $\Omega$ é um domínio suave em $\mathbb{R}^{3}$. O objetivo deste trabalho é estudar o intervalo maximal de existência no tempo de solução para o sistema acima em domínios tridimensionais finos, isto é, quando uma dimensão é pequena quando comparada as demais. Em outras palavras, consideraremos um domínio do tipo $\Omega_{\epsilon}=$ $\omega \times(0, \epsilon) \subset \mathbb{R}^{3}$, sendo $\omega \subset \mathbb{R}^{2}$ um domíno regular e $0<\epsilon<1$.

O estudo de solução global no tempo para domínios finos foi iniciado por Raugel e Sell (1989), os quais consideraram uma dilatação no domínio para encontrar as equações de Navier-Stokes definidas em $\omega \times(0,1)$ fixo. Diferentemente, o artigo de Temam e Ziane (1996), não usa a dilatação e sim, trabalha diretamente em $\Omega_{\epsilon}$. Além disso, consideramos combinações entre condições de fronteira, as quais influenciam fortemente no comportamento das soluções.

Nosso trabalho está estruturado em duas seções principais; na primeira seção, deduzimos uma formulação matemática específica para domínios finos, como as combinações entre as condições de fronteira de Dirichlet, periódica e livre, e a interferência dessas na definição dos espaços funcionais $H$ e $V$. Outra importante ferramenta usada nesta seção é o operador média integral na direção fina, definido de acordo com a condição de fronteira a ser utilizado. Por fim, apresentamos algumas desigualdades clássicas, como a de Poincaré, além de outras essencialmente importantes.

Na segunda seção, é feita uma formulação fraca para as equações de Navier-Stokes, baseando-se no operador média integral, além de estimativas a priori para o mesmo. Tais estimativas tornam-se uma importante ferramenta para os resultados acerca do intervalo maximal de existência de solução no tempo, estes dependendo da condição de fronteira considerada.

\section{Introdução ao estudo em domínios finos}

No desenvolver deste trabalho, usamos notações e resultados usuais no estudo de equações diferenciais parciais e em análise funcional, baseando-se principalmente em Evans (2010), Adams e Fournier (2003), Brezis (1983) e Rudin (1986). No que segue, $\Omega$ é um aberto limitado do $\mathbb{R}^{3}$ e $L^{p}(\Omega), 1 \leq p<\infty$, é o espaço das funções mensuráveis $g: \Omega \rightarrow \mathbb{R}$ tais que

$$
|g|_{L^{p}(\Omega)}=\left(\int_{\Omega}|g(x)|^{p} d x\right)^{1 / p}<\infty .
$$

e $H^{1}(\Omega)$ é o espaço de Sobolev no qual todas as derivadas parciais de primeira ordem, com relação a todas variáveis, pertencem à $L^{2}(\Omega)$.

Considera-se também os espaços funcionais:

1. $\Lambda=\left\{u \in C_{\mathcal{C}}^{\infty}(\Omega) ; \operatorname{div} u=0\right\}$;

2. $H$ é o fecho de $\Lambda$ em $L^{2}\left(\Omega_{\epsilon}\right)$, munido da norma

$$
|u|_{\epsilon}=\left(\int_{\Omega_{\epsilon}}|u|^{2} d x\right)^{1 / 2}
$$

3. $V$ é o fecho de $\Lambda$ em $H_{0}^{1}\left(\Omega_{\epsilon}\right)$, munido da norma

$$
\|u\|_{\epsilon}=|\nabla u|_{\epsilon}=\left(\int_{\Omega_{\epsilon}}|\nabla u|^{2} d x\right)^{1 / 2}
$$


Os produtos internos de $H$ e $V$ são denotados por $(\cdot, \cdot)_{\epsilon}$ e $((\cdot, \cdot))_{\epsilon}$, respectivamente. A fórmula trilinear $b_{\epsilon}$ é dada por

$$
b_{\epsilon}(u, v, w)=(u \cdot \nabla v, w)_{\epsilon}=\sum_{i, j=1}^{3} \int_{\Omega_{\epsilon}} u_{i} \frac{\partial v_{j}}{\partial x_{i}} w_{j} d x
$$

onde $u, v, w \in H^{1}(\Omega)$. Ainda, faz-se o uso do Operador de Stokes:

Definição 2.1. Seja $P: L^{2}\left(\Omega_{\epsilon}\right) \rightarrow H_{\epsilon}$ o operador projeção. Define-se o operador de Stokes, denotado por $A_{\epsilon}$, por

$$
\begin{aligned}
A_{\epsilon}: V_{\epsilon} \cap H^{2}\left(\Omega_{\epsilon}\right) \subset H & \longrightarrow H \\
u & \longmapsto A_{\epsilon} u=-P(\Delta u)
\end{aligned}
$$

Bastante utilizado são as potências do Operador de Stokes, em particular, $A_{\epsilon}^{1 / 2}$. Quando usamos esta potência, o domínio do operador coincide com $V$, ou seja, $D\left(A_{\epsilon}\right)=V$. Sendo assim, se $u \in V$, então

$$
\|u\|_{\epsilon}=\left|A_{\epsilon}^{1 / 2} u\right|_{\epsilon}
$$

Para mais detalhes sobre este operador, veja Foias et al. (2002).

Seja $\Omega_{\epsilon}$ um domínio fino, isto é, $\Omega_{\epsilon}=\omega \times(0, \epsilon)$, onde $0<\epsilon<1$ e $\omega \subset \mathbb{R}^{2}$ é suave. O objetivo deste trabalho é estudar o intervalo maximal de existência de soluções $u(x, t)$ no tempo do sistema

$$
\begin{cases}u_{t}+u \cdot \nabla u-\mu \Delta u+\nabla p=f, & \text { em } \Omega_{\epsilon} \times(0, \infty) \\ \operatorname{div} u=0, & \text { em } \Omega_{\epsilon} \times(0, \infty) \\ u(x, 0)=u_{0}(x), & \text { em } x \in \Omega_{\epsilon},\end{cases}
$$

onde

(i) $u(x, t)=\left(u_{1}(x, t), u_{2}(x, t), u_{3}(x, t)\right)$ é o campo de velocidades no ponto $x=\left(x_{1}, x_{2}, x_{3}\right)$ e no instante $t$

(ii) $p: \Omega_{\epsilon} \times(0, T) \rightarrow \mathbb{R}, T>0$, é a pressão;

(iii) $\mu>0$ é a viscosidade do sistema e;

(iv) $u_{0}(x)=\left(u_{0}^{1}(x), u_{0}^{2}(x), u_{0}^{3}(x)\right)$ é a velocidade inicial dada.

Neste trabalho, supõe-se que

$$
u_{0} \in H \text { ou } V \text { e } f \in L^{\infty}(0,+\infty, H)
$$

Para isso, serão necessárias algumas definições iniciais com relação a fronteira de $\Omega_{\epsilon}$ e aos espaços $V$ e $H$, os quais serão definidos de acordo com a condição de fronteira em questão.

\subsection{Condições de Fronteira}

No que segue, a fronteira $\partial \Omega_{\epsilon}$ de $\Omega_{\epsilon}$ será denotada por $\partial \Omega_{\epsilon}=\Gamma_{t} \cup \Gamma_{b} \cup \Gamma_{l}$, onde

$$
\Gamma_{t}=\omega \times\{\epsilon\}, \quad \Gamma_{b}=\omega \times\{0\}, \text { e } \Gamma_{l}=\partial \omega \times(0, \epsilon) .
$$

Para a formulação do problema, faz-se algumas combinações entre certas condições para $\partial \Omega_{\epsilon}$, a saber, as condições de Dirichlet, periódica e de fronteira livre.

Mais precisamente, as combinações usadas são:

1. A condição de fronteira livre em $\Gamma_{t} \cup \Gamma_{b}$ e periódica em $\Gamma_{l}$, denotada por (FP). Considera-se, neste caso, $\omega=\left(0, l_{1}\right) \times\left(0, l_{2}\right), l_{1}, l_{2}>0$,

$$
u_{3}=0 \quad \text { e } \quad \frac{\partial u_{1}}{\partial x_{3}}=\frac{\partial u_{2}}{\partial x_{3}}=0 \quad \text { em } \quad \Gamma_{t} \cup \Gamma_{b},
$$

e $u_{i}$ é periódica nas direções $x_{1}$ e $x_{2}$ com período $l_{1}$ e $l_{2}$, respectivamente, para $i=1,2,3$. Além disso,

$$
\int_{\Omega_{\epsilon}} u_{0}^{j}(x) d x=\int_{\Omega_{\epsilon}} f_{j}(x, t) d x=0, \quad j=1,2 .
$$

2. A condição de fronteira livre em $\partial \Omega_{\epsilon}$, denotada por (FF). Neste caso, se $\mathbf{n}$ é o vetor normal apontado para fora de $\partial \Omega_{\epsilon}$, então

$$
u \cdot \mathbf{n}=0, \quad \operatorname{rot} u \times \mathbf{n}=0 \text { em } \partial \Omega_{\epsilon} .
$$

A primeira relação acima indica que a velocidade é sempre perpendicular ao vetor normal, isto é, acompanha a superfície da fronteira.

3. A condição de Dirichlet em $\partial \Omega_{\epsilon}$, denotada por (DD), ou seja,

$$
u(x, t)=0 \text { em } \partial \Omega_{\epsilon} .
$$

4. A condição de Dirichlet em $\Gamma_{t} \cup \Gamma_{b}$ e periódica em $\Gamma_{l}$, denotada por (DP). Neste caso,

$$
u(x, t)=0 \text { em } \Gamma_{t} \cup \Gamma_{b}
$$

e $u$ é periódica em $\Gamma_{l}$. Além disso,

$$
\int_{\Omega_{\varepsilon}} u_{0}^{3} d x=\int_{\Omega_{\epsilon}} f_{3}(x, t) d x=0 .
$$

Observe que os espaços $H$ e $V$ são influenciados por estas condições de fronteiras. Sendo assim, quando não houver diferença nas demonstrações, utilizaremos a notação para estes espaços por $H_{\epsilon}$ e $V_{\epsilon}$.

\subsection{Os operadores $\tilde{M}_{\epsilon}$ e $\tilde{N}_{\epsilon}$}

Seja $\phi \in L^{2}\left(\Omega_{\epsilon}\right)$ uma função escalar. O operador média integral na direção fina é definido por:

$$
M_{\epsilon}: L^{2}\left(\Omega_{\epsilon}\right) \quad \longrightarrow \quad L^{2}\left(\Omega_{\epsilon}\right)
$$




$$
\phi \longmapsto M_{\epsilon} \phi=\frac{1}{\epsilon} \int_{0}^{\epsilon} \phi\left(x_{1}, x_{2}, s\right) d s .
$$

Note que apesar de $\phi$ ser uma função de três variáveis, $M_{\epsilon}$ possui apenas duas, a saber, $x_{1}$ e $x_{2}$, visto que a integral em (1) depende apenas da terceira componente de $\phi$. Define-se, também, o operador $N_{\epsilon}$ por

$$
N_{\epsilon} \phi\left(x_{1}, x_{2}, x_{3}\right)=\phi\left(x_{1}, x_{2}, x_{3}\right)-M_{\epsilon} \phi\left(x_{1}, x_{2}\right) \text {. }
$$

Portanto,

$$
N_{\epsilon}+M_{\epsilon}=I_{L^{2}\left(\Omega_{\epsilon}\right)} \cdot
$$

O operador $\tilde{M}_{\epsilon}$ é definido para funções $u=\left(u_{1}, u_{2}, u_{3}\right) \in$ $\mathbb{L}^{2}\left(\Omega_{\epsilon}\right)$, isto é,

$$
\begin{aligned}
\tilde{M}_{\epsilon}: \mathbb{L}^{2}\left(\Omega_{\epsilon}\right) & \longrightarrow \mathbb{L}^{2}\left(\Omega_{\epsilon}\right) \\
u & \longmapsto \tilde{M}_{\epsilon} u=\left(M_{\epsilon} u_{1}, M_{\epsilon} u_{2}, M_{\epsilon} u_{3}\right)
\end{aligned}
$$

Dependendo da condição de fronteira, define-se:

(i) Para (FF) e (FP), $\tilde{M}_{\epsilon} u=\left(M_{\epsilon} u_{1}, M_{\epsilon} u_{2}, 0\right)$.

(ii) Para (DD) e (DP), $\tilde{M}_{\epsilon} u=0$.

Analogamente, define-se o operador $\tilde{N}_{\epsilon}$ por $\tilde{N}_{\epsilon} u=$ $u-\tilde{M}_{\epsilon} u$, ou seja, $\tilde{N}_{\epsilon}+\tilde{M}_{\epsilon}=I_{\mathbb{L}^{2}\left(\Omega_{\epsilon}\right)}$.

Um fato interessante sobre os operadores definidos acima é que todos são projeções, isto é,

$$
M_{\epsilon}^{2}=M_{\epsilon}, N_{\epsilon}^{2}=N_{\epsilon}, \tilde{M}_{\epsilon}^{2}=\tilde{M}_{\epsilon}, \tilde{N}_{\epsilon}^{2}=\tilde{N}_{\epsilon} .
$$

De fato, seja $\phi \in L^{2}\left(\Omega_{\epsilon}\right)$. Então,

$$
M_{\epsilon}^{2}(\phi)=\left(M_{\epsilon} \circ M_{\epsilon}\right)(\phi)=M_{\epsilon}\left(M_{\epsilon} \phi\right) .
$$

Assim,

$$
\begin{aligned}
M_{\epsilon}\left(M_{\epsilon} \phi\right) & =\int_{0}^{\epsilon} \frac{1}{\epsilon} M_{\epsilon} \phi\left(x_{1}, x_{2}\right) d x_{3} \\
& =\frac{M_{\epsilon} \phi\left(x_{1}, x_{2}\right)}{\epsilon} \int_{0}^{\epsilon} d x_{3} \\
& =M_{\epsilon} \phi\left(x_{1}, x_{2}\right) .
\end{aligned}
$$

Para $N_{\epsilon}$, tem-se

$$
\begin{aligned}
N_{\epsilon}^{2}(\phi)=N_{\epsilon}\left(N_{\epsilon} \phi\right) & =N_{\epsilon}\left(\phi-M_{\epsilon} \phi\right) \\
& =N_{\epsilon} \phi-N_{\epsilon}\left(M_{\epsilon} \phi\right) \\
& =\phi-M_{\epsilon} \phi-\left(M_{\epsilon} \phi-M_{\epsilon}\left(M_{\epsilon} \phi\right)\right) \\
& =\phi-M_{\epsilon} \phi-\left(M_{\epsilon} \phi-M_{\epsilon} \phi\right) \\
& =\phi-M_{\epsilon} \phi=N_{\epsilon} \phi
\end{aligned}
$$

com $\phi \in L^{2}\left(\Omega_{\epsilon}\right)$. Analogamente mostra-se para $\tilde{M}_{\epsilon}$ e $\tilde{N}_{\epsilon}$. Além disso, é pertinente observar que, independente da condição de fronteira, cada componente de $\tilde{N}_{\epsilon} u$ satisfaz uma das seguintes situações:

$$
\begin{aligned}
& N_{\epsilon} u_{j} \equiv 0 \text { em } \Gamma_{t} \cup \Gamma_{b} \quad \text { ou } \\
& \quad \int_{0}^{\epsilon} N_{\epsilon} u_{j} d x_{3}=0, \quad \text { para } j=1,2,3 .
\end{aligned}
$$

O seguinte resultado reúne algumas propriedades para os operadores $M_{\epsilon}, N_{\epsilon}, \tilde{M}_{\epsilon}, \tilde{N}_{\epsilon}$, as quais serão úteis na demonstração do lema 2.1.
Proposição 2.1. Vale que:

(i) $M_{\epsilon}$ é a projeção ortogonal de $L^{2}\left(\Omega_{\epsilon}\right)$ em $L^{2}(\omega)$.

(ii) $M_{\epsilon} N_{\epsilon}=0$ e $\tilde{M}_{\epsilon} \tilde{N}_{\epsilon}=0$.

(iii) $\tilde{M}_{\epsilon} \nabla^{\prime}=\nabla^{\prime} M_{\epsilon}, \tilde{N}_{\epsilon} \nabla^{\prime}=\nabla^{\prime} N_{\epsilon}$.

(iv) Se $\phi \in H^{k}\left(\Omega_{\epsilon}\right)$ então $M_{\epsilon} \phi \in H^{k}(\omega)$ e $N_{\epsilon} \phi \in$ $H^{k}\left(\Omega_{\epsilon}\right), \forall k \geq 0$.

(v) A condição de fronteira para $\tilde{M}_{\epsilon}$ em $\partial \omega$ é a mesma de $u \in \mathbb{L}^{2}\left(\Omega_{\epsilon}\right)$ em $\partial \omega \times(0, \epsilon)$.

(vi) Os operadores $\tilde{M}_{\epsilon}$ e $\tilde{N}_{\epsilon}$ são auto-adjuntos.

\section{Demonstração:}

(i) Dado $\phi \in L^{2}\left(\Omega_{\epsilon}\right)$ tem-se, para todo $\theta \in L^{2}(\omega)$,

$$
\begin{aligned}
\left(\phi-M_{\epsilon} \phi, \theta\right)_{L^{2}\left(\Omega_{\epsilon}\right)} & =\int_{\Omega_{\epsilon}}\left[\phi-M_{\epsilon} \phi\right] \theta d x \\
& =\int_{\Omega_{\epsilon}} \phi \theta-M_{\epsilon} \phi \theta d x \\
& =\int_{\Omega_{\epsilon}} \phi \theta d x-\int_{\Omega_{\epsilon}} M_{\epsilon} \phi \theta d x(3)
\end{aligned}
$$

Resolvendo a primeira integral de (3), obtêm-se

$$
\int_{\Omega_{\epsilon}} \phi \theta d x=\epsilon \int_{\omega} \theta\left(M_{\epsilon} \phi\right) d x .
$$

Da segunda integral de (3),

$$
\int_{\Omega_{\epsilon}} M_{\epsilon} \phi \theta d x=\epsilon \int_{\omega} \theta\left(M_{\epsilon} \phi\right) d x .
$$

Comparando (4) e (5), segue que

$$
\left(\phi-M_{\epsilon} \phi, \theta\right)_{L^{2}\left(\Omega_{\epsilon}\right)}=0,
$$

ou seja, $M_{\epsilon}$ é a projeção ortogonal de $L^{2}\left(\Omega_{\epsilon}\right)$ em $L^{2}(\omega)$.

(ii) Tem-se, para $\phi \in L^{2}\left(\Omega_{\epsilon}\right)$,

$$
\begin{aligned}
M_{\epsilon} N_{\epsilon} & =M_{\epsilon}\left(N_{\epsilon} \phi\right) \\
& =M_{\epsilon}\left(\phi-M_{\epsilon} \phi\right) \\
& =M_{\epsilon} \phi-M_{\epsilon}\left(M_{\epsilon} \phi\right) \\
& =M_{\epsilon} \phi-M_{\epsilon} \phi=0 .
\end{aligned}
$$

Para $\tilde{M}_{\epsilon} \tilde{N}_{\epsilon}$ a demonstração é análoga.

(iii) Seja $\phi \in L^{2}\left(\Omega_{\epsilon}\right)$. Então,

$$
\begin{aligned}
\tilde{M}_{\epsilon} \nabla^{\prime} & =\tilde{M}_{\epsilon}\left(\nabla^{\prime} \phi\right) \\
& =\tilde{M}_{\epsilon}\left(\frac{\partial \phi}{\partial x_{1}}, \frac{\partial \phi}{\partial x_{2}}, 0\right) \\
& =\left(M_{\epsilon}\left(\frac{\partial \phi}{\partial x_{1}}\right), M_{\epsilon}\left(\frac{\partial \phi}{\partial x_{2}}\right), 0\right) .
\end{aligned}
$$


Pelo Teorema da Convergência Dominada, pode-se inverter a integração pela derivação em cada coordenada do último vetor da expressão acima. Assim,

$$
\begin{aligned}
\left(M_{\epsilon}\left(\frac{\partial \phi}{\partial x_{1}}\right), M_{\epsilon}\left(\frac{\partial \phi}{\partial x_{2}}\right), 0\right) & = \\
\left(\frac{\partial}{\partial x_{1}} M_{\epsilon} \phi, \frac{\partial}{\partial x_{2}} M_{\epsilon} \phi, 0\right) & =\nabla^{\prime} M_{\epsilon} \phi
\end{aligned}
$$

Logo, $\tilde{M}_{\epsilon}\left(\nabla^{\prime} \phi\right)=\nabla^{\prime} M_{\epsilon} \phi$. Para $\tilde{N}_{\epsilon} \nabla^{\prime}$, tem-se

$$
\begin{aligned}
\left(\tilde{N}_{\epsilon} \nabla^{\prime}\right)(\phi) & =\tilde{N}_{\epsilon}\left(\nabla^{\prime} \phi\right)=\tilde{N}\left(\frac{\partial \phi}{\partial x_{1}}, \frac{\partial \phi}{\partial x_{2}}, 0\right) \\
& =\left(N_{\epsilon}\left(\frac{\partial \phi}{\partial x_{1}}\right), N_{\epsilon}\left(\frac{\partial \phi}{\partial x_{2}}\right), 0\right) \\
= & \left(\frac{\partial \phi}{\partial x_{1}}-M_{\epsilon}\left(\frac{\partial \phi}{\partial x_{1}}\right), \frac{\partial \phi}{\partial x_{2}}-M_{\epsilon}\left(\frac{\partial \phi}{\partial x_{2}}\right), 0\right) .
\end{aligned}
$$

Novamente, pelo Teorema da Convergência dominada,

$$
\begin{array}{r}
\left(\frac{\partial \phi}{\partial x_{1}}-M_{\epsilon}\left(\frac{\partial \phi}{\partial x_{1}}\right), \frac{\partial \phi}{\partial x_{2}}-M_{\epsilon}\left(\frac{\partial \phi}{\partial x_{2}}\right), 0\right)= \\
\left(\frac{\partial}{\partial x_{1}} N_{\epsilon} \phi, \frac{\partial}{\partial x_{2}} N_{\epsilon} \phi, 0\right)= \\
\nabla^{\prime} N_{\epsilon} \phi .
\end{array}
$$

Logo, $\tilde{N}_{\epsilon}\left(\nabla^{\prime} \phi\right)=\nabla^{\prime} N_{\epsilon} \phi$

(iv) Seja $\phi \in H^{k}\left(\Omega_{\epsilon}\right)$. Para $k=0$, tem-se

$$
\begin{array}{r}
\int_{\omega}\left|M_{\epsilon} \phi\left(x_{1}, x_{2}\right)\right|^{2} d x_{1} d x_{2}= \\
\int_{\omega}\left|\frac{1}{\epsilon} \int_{0}^{\epsilon} \phi\left(x_{1}, x_{2}, s\right) d s\right|^{2} d x_{1} d x_{2} .
\end{array}
$$

Mas, pela Desigualdade de Hölder, para $p=q=2$,

$$
\int_{0}^{\epsilon}|\phi| d s \leq\left(\int_{0}^{\epsilon} 1 d s\right)^{1 / 2}\left(\int_{0}^{\epsilon}|\phi|^{2} d s\right)^{1 / 2}
$$

Logo,

$$
\left(\int_{0}^{\epsilon}|\phi| d s\right)^{2} \leq \epsilon \int_{0}^{\epsilon}|\phi|^{2} d s
$$

De (6) e (7), obtem-se

$$
\begin{array}{r}
\int_{\omega}\left|\frac{1}{\epsilon} \int_{0}^{\epsilon} \phi d s\right|^{2} d x_{1} d x_{2} \leq \\
\int_{\omega}\left(\frac{1}{\epsilon} \int_{0}^{\epsilon}|\phi| d s\right)^{2} d x_{1} d x_{2} \leq \\
\int_{\omega} \frac{1}{\epsilon}\left(\epsilon \int_{0}^{\epsilon}|\phi|^{2} d s\right) d x_{1} d x_{2}= \\
\epsilon\|\phi\|_{L^{2}\left(\Omega_{\epsilon}\right)}^{2} .
\end{array}
$$

Assim, $\left\|M_{\epsilon} \phi\right\|_{L^{2}(\omega)}^{2} \leq \epsilon\|\phi\|_{L^{2}\left(\Omega_{\epsilon}\right)}^{2}$. Como $\phi \in H^{k}\left(\Omega_{\epsilon}\right)$, segue que $\left\|M_{\epsilon} \phi\right\|_{L^{2}(\omega)}^{2}$ é finito e portanto $M_{\epsilon} \phi \in H^{k}(\omega)$.
Além disso, já que $M_{\epsilon} \phi \in H^{k}(\omega)$ e $\phi \in H^{k}\left(\Omega_{\epsilon}\right)$, segue que $N_{\epsilon} \phi=\phi-M_{\epsilon} \phi \in H^{k}\left(\Omega_{\epsilon}\right)$.

(v) Suponha que $u$ satisfaz (DD). Então, $u \equiv 0 \mathrm{em}$ $\Gamma_{l}$, isto é, $u_{i} \equiv 0$ para $i=1,2,3 \mathrm{em} \partial \omega \times(0, \epsilon)$. Assim, como $\tilde{M}_{\epsilon} u=\left(M_{\epsilon} u_{1}, M_{\epsilon} u_{2}, M_{\epsilon} u_{3}\right)$, tem-se que $M_{\epsilon} u_{i} \equiv 0$ para $i=1,2,3$, ou seja, $\tilde{M}_{\epsilon} u \equiv 0$ em $\partial \omega$.

Se $u$ satisfaz (FP), $u$ é periódica nas direções $x_{1}$ e $x_{2}$ com período $l_{1}, l_{2}>0$, ou seja, para todo $i=1,2,3$, temse $u_{i}\left(x_{1}+l_{1}, x_{2}+l_{2}, x_{3}\right)=u_{i}\left(x_{1}, x_{2}, x_{3}\right)$. Assim, para todo $i=1,2,3$,

$$
\begin{aligned}
& M_{\epsilon} u_{i}\left(x_{1}+l_{1}, x_{2}+l_{2}\right)= \\
& \frac{1}{\epsilon} \int_{0}^{\epsilon} u_{i}\left(x_{1}+l_{1}, x_{2}+l_{2}, s\right) d s= \\
& \frac{1}{\epsilon} \int_{0}^{\epsilon} u_{i}\left(x_{1}, x_{2}, s\right) d s=M_{\epsilon} u_{i}\left(x_{1}, x_{2}\right) \text {, }
\end{aligned}
$$

isto é, $\tilde{M}_{\epsilon} u$ é periódica nas direções $x_{1}$ e $x_{2}$.

A condição (FF) implica que $u \cdot \mathbf{n}=0$ e $\operatorname{rot}(u \times \mathbf{n})=$ 0 em $\partial \Omega_{\epsilon}$. Logo,

$$
\begin{gathered}
\tilde{M}_{\epsilon} u\left(x_{1}, x_{2}\right)=\left(M_{\epsilon} u_{1}, M_{\epsilon} u_{2}, M_{\epsilon} u_{3}\right)= \\
\left(\frac{1}{\epsilon} \int_{0}^{\epsilon} u_{1} d s, \frac{1}{\epsilon} \int_{0}^{\epsilon} u_{2} d s, \frac{1}{\epsilon} \int_{0}^{\epsilon} u_{3} d s\right)= \\
\frac{1}{\epsilon} \int_{0}^{\epsilon}\left(u_{1}, u_{2}, u_{3}\right) d s .
\end{gathered}
$$

Assim,

$$
\tilde{M}_{\epsilon} u \cdot \mathbf{n}=\frac{1}{\epsilon} \int_{0}^{\epsilon}\left(u_{1}, u_{2}, u_{3}\right) \cdot \mathbf{n} d s=0 .
$$

Além disso, $\left(\tilde{M}_{\epsilon} u\right) \times \mathbf{n}=\tilde{M}_{\epsilon}(u \times \mathbf{n})$ e

$$
\begin{aligned}
\operatorname{rot}\left(\left(\tilde{M}_{\epsilon} u\right) \times \mathbf{n}\right) & =\operatorname{rot}\left(\tilde{M}_{\epsilon}(u \times \mathbf{n})\right) \\
& =\tilde{M}_{\epsilon}(\operatorname{rot}(u \times \mathbf{n}))=0,
\end{aligned}
$$

quando $\left(x_{1}, x_{2}\right) \in \partial \omega$ e $x_{3} \in(0, \epsilon)$.

Portanto, $\tilde{M}_{\epsilon} u$ satisfaz (FF).

(vi) Sejam $u, v \in \mathbb{L}^{2}\left(\Omega_{\epsilon}\right)$. Então,

$$
\begin{aligned}
\left(\tilde{M}_{\epsilon} u, v\right)_{\epsilon} & =\int_{\Omega_{\epsilon}}\left(\tilde{M}_{\epsilon} u\right) v d x \\
& =\int_{0}^{\epsilon} \int_{\omega}\left(\tilde{M}_{\epsilon} u\right) v d x_{1} d x_{2} d x_{3} \\
& =\int_{\omega} \tilde{M}_{\epsilon} u\left[\int_{0}^{\epsilon} v d x_{3}\right] d x_{1} d x_{2} \\
& =\int_{\omega}\left[\frac{1}{\epsilon} \int_{0}^{\epsilon} u d s \int_{0}^{\epsilon} v d x_{3}\right] d x_{1} d x_{2} \\
& =\int_{\omega}\left[\int_{0}^{\epsilon} u d s\right] \tilde{M}_{\epsilon} v d x_{1} d x_{2} \\
& =\int_{\Omega_{\epsilon}} u\left(\tilde{M}_{\epsilon} v\right) d x=\left(u, \tilde{M}_{\epsilon} v\right)_{\epsilon} .
\end{aligned}
$$

Ainda,

$$
\left(\tilde{N}_{\epsilon} u, v\right)_{\epsilon}=\left(u-\tilde{M}_{\epsilon} u, v\right)_{\epsilon}
$$




$$
\begin{aligned}
& =(u, v)_{\epsilon}-\left(\tilde{M}_{\epsilon} u, v\right)_{\epsilon} \\
& =(u, v)_{\epsilon}-\left(u, \tilde{M}_{\epsilon} v\right)_{\epsilon} \\
& =\left(u, v-\tilde{M}_{\epsilon} v\right)_{\epsilon}=\left(u, \tilde{N}_{\epsilon} v\right)_{\epsilon}
\end{aligned}
$$

Os resultados do lema a seguir independem da condição de fronteira escolhida e por isso estas serão omitidas.

Lema 2.1. Sejam $u, v \in \mathbb{H}^{1}\left(\Omega_{\epsilon}\right)$. Então:

(i) $\int_{\Omega_{\epsilon}} \nabla \tilde{N}_{\epsilon} u \cdot \nabla \tilde{M}_{\epsilon} v d x=0$

(ii) $|u|_{\epsilon}^{2}=\left|\tilde{M}_{\epsilon} u\right|_{\epsilon}^{2}+\left|\tilde{N}_{\epsilon} u\right|_{\epsilon}^{2}$ e $\left\|\left.u\right|_{\epsilon} ^{2}=\right\| \tilde{M}_{\epsilon} u \|_{\epsilon}^{2}+$ $\left\|\tilde{N}_{\epsilon} u\right\|_{\epsilon}^{2}$

(iii) Se $v \in V_{\epsilon}$ então $\tilde{M}_{\epsilon} v \in V_{\epsilon}$ e $\tilde{N}_{\epsilon} v \in V_{\epsilon}$

(iv) $b_{\epsilon}\left(u, u, \tilde{M}_{\epsilon} v\right)=b_{\epsilon}\left(\tilde{M}_{\epsilon} u, \tilde{M}_{\epsilon} u, \tilde{M}_{\epsilon} v\right)$

$+b_{\epsilon}\left(\tilde{N}_{\epsilon} u, \tilde{N}_{\epsilon} u, \tilde{M}_{\epsilon} v\right) e$

$b_{\epsilon}\left(u, u, \tilde{N}_{\epsilon} v\right)=b_{\epsilon}\left(\tilde{N}_{\epsilon} u, \tilde{M}_{\epsilon} u, \tilde{N}_{\epsilon} v\right)$

$+b_{\epsilon}\left(\tilde{M}_{\epsilon} u, \tilde{N}_{\epsilon} u, \tilde{N}_{\epsilon} v\right)+b_{\epsilon}\left(\tilde{N}_{\epsilon} u, \tilde{N}_{\epsilon} u, \tilde{N}_{\epsilon} v\right)$, onde $u, v \in V_{\epsilon}$.

(v) Para todo $u \in D\left(A_{\epsilon}\right)$, tem-se

$$
\Delta \tilde{N}_{\epsilon} u=\tilde{N}_{\epsilon} \Delta u \text { e } \Delta \tilde{M}_{\epsilon} u=\tilde{M}_{\epsilon} \Delta u
$$

\section{Demonstração:}

(i) Sejam $u, v \in \mathbb{H}^{1}\left(\Omega_{\epsilon}\right)$. Tem-se

$$
\begin{array}{r}
\int_{\Omega_{\epsilon}} \nabla \tilde{N}_{\epsilon} u \cdot \nabla \tilde{M}_{\epsilon} v d x \\
\int_{\Omega_{\epsilon}}\left(\nabla N_{\epsilon} u_{1} \nabla M_{\epsilon} v_{1}+\nabla N_{\epsilon} u_{2} \nabla M_{\epsilon} v_{2}\right. \\
\left.+\nabla N_{\epsilon} u_{3} \nabla M_{\epsilon} v_{3}\right) d x
\end{array}
$$

Note que a expressão acima é igual a

$$
\begin{aligned}
\int_{\Omega_{\epsilon}}\left(\nabla^{\prime} N_{\epsilon} u_{1} \cdot \nabla^{\prime} M_{\epsilon} v_{1}\right. & +\frac{\partial N_{\epsilon} u_{1}}{\partial x_{3}} \frac{\partial M_{\epsilon} v_{1}}{\partial x_{3}}+ \\
\nabla^{\prime} N_{\epsilon} u_{2} \cdot \nabla^{\prime} M_{\epsilon} v_{2} & +\frac{\partial N_{\epsilon} u_{2}}{\partial x_{3}} \frac{\partial M_{\epsilon} v_{2}}{\partial x_{3}}+ \\
& +\nabla^{\prime} N_{\epsilon} u_{3} \cdot \nabla^{\prime} M_{\epsilon} v_{3}+ \\
& \left.+\frac{\partial N_{\epsilon} u_{3}}{\partial x_{3}} \frac{\partial M_{\epsilon} v_{3}}{\partial x_{3}}\right) d x
\end{aligned}
$$

Como $M_{\epsilon} v_{i}$ não dependem de $x_{3}$, pois $N_{\epsilon} u_{i}$ depende de $x_{3}$, segue que $\frac{\partial M_{\epsilon} v_{i}}{\partial x_{3}} \equiv 0$, para $i=1,2,3$ e, além disso, pela propriedade (iii) da proposição anterior, $\nabla^{\prime} N_{\epsilon} u_{i}$. $\nabla^{\prime} M_{\epsilon} v_{i}=\tilde{N}_{\epsilon} \nabla^{\prime} u_{i} \cdot \tilde{M}_{\epsilon} \nabla^{\prime} v_{i}, i=1,2,3$. Logo, (8) é igual à

$$
\begin{gathered}
\int_{\Omega_{\epsilon}}\left(\tilde{N}_{\epsilon} \nabla^{\prime} u_{1} \cdot \tilde{M}_{\epsilon} \nabla^{\prime} v_{1}+\tilde{N}_{\epsilon} \nabla^{\prime} u_{2} \cdot \tilde{M}_{\epsilon} \nabla^{\prime} v_{2}+\right. \\
\left.\tilde{N}_{\epsilon} \nabla^{\prime} u_{3} \cdot \tilde{M}_{\epsilon} \nabla^{\prime} v_{3}\right) d x
\end{gathered}
$$

Observe que o conjunto

$$
A=\left\{\frac{1}{\epsilon} \int_{0}^{\epsilon} \phi\left(x_{1}, x_{2}, s\right) d s, \phi \in L^{2}\left(\Omega_{\epsilon}\right)\right\} \subset L^{2}(\omega)
$$

é fechado. De fato, tomando uma sequência de funções $\left(\phi_{n}\right) \subset A$ tal que $\phi_{n} \rightarrow \phi$, onde $\phi \in L^{2}(\omega)$, basta escolher $\theta\left(x_{1}, x_{2}, x_{3}\right) \in L^{2}\left(\Omega_{\epsilon}\right)$ de modo que $\phi=\frac{1}{\epsilon} \int_{0}^{\epsilon} \theta d s$ e assim $\phi \in A$. Portanto, pela propriedade (i) da proposição anterior, pode-se decompor $L^{2}\left(\Omega_{\epsilon}\right)$ como a soma direta de $M_{\epsilon}\left(L^{2}\left(\Omega_{\epsilon}\right)\right)$ e $N_{\epsilon}\left(L^{2}\left(\Omega_{\epsilon}\right)\right)$ da mesma maneira que $\mathbb{L}^{2}\left(\Omega_{\epsilon}\right)$ é soma direta de $\tilde{M}_{\epsilon}\left(L^{2}\left(\Omega_{\epsilon}\right)\right)$ e $\tilde{N}_{\epsilon}\left(L^{2}\left(\Omega_{\epsilon}\right)\right)$. Logo,

$$
\int_{\Omega_{\epsilon}} \tilde{N}_{\epsilon} \nabla^{\prime} u_{i} \cdot \tilde{M}_{\epsilon} \nabla^{\prime} v_{i} d x=0,
$$

para todo $i=1,2,3$ e a integral em (9) é nula, ou seja,

$$
\int_{\Omega_{\epsilon}} \nabla \tilde{N}_{\epsilon} u \cdot \nabla \tilde{M}_{\epsilon} v d x=0 .
$$

(ii) No item anterior, foi provado que o conjunto $A$ é fechado e portanto, pode-se escrever $u \in \mathbb{L}^{2}\left(\Omega_{\epsilon}\right)$ como a soma dos vetores ortogonais $\tilde{M}_{\epsilon} u$ e $\tilde{N}_{\epsilon} u$. Assim,

$$
\begin{aligned}
|u|_{\epsilon}^{2} & =\int_{\Omega_{\epsilon}}|u|^{2} d x=\int_{\Omega_{\epsilon}}\left|\left(\tilde{M}_{\epsilon} u+\tilde{N}_{\epsilon} u\right)\right|^{2} d x \\
& =\int_{\Omega_{\epsilon}}\left|\left(\tilde{M}_{\epsilon} u+\tilde{N}_{\epsilon} u\right) \cdot\left(\tilde{M}_{\epsilon} u+\tilde{N}_{\epsilon} u\right)\right| d x \\
& =\left|\tilde{M}_{\epsilon} u\right|_{\epsilon}^{2}+\left|\tilde{N}_{\epsilon} u\right|_{\epsilon}^{2}+\underbrace{2 \int_{\Omega_{\epsilon}} \tilde{M}_{\epsilon} u \cdot \tilde{N}_{\epsilon} u d x .}_{=0 \text { pois } \tilde{M}_{\epsilon} u \perp \tilde{N}_{\epsilon} u}
\end{aligned}
$$

Logo,

$$
|u|_{\epsilon}^{2}=\left|\tilde{M}_{\epsilon} u\right|_{\epsilon}^{2}+\left|\tilde{N}_{\epsilon} u\right|_{\epsilon}^{2} .
$$

Além disso, vale que

$$
\begin{aligned}
\|u\|_{\epsilon}^{2} & =\int_{\Omega_{\epsilon}}|\nabla u|^{2} d x=\int_{\Omega_{\epsilon}}\left|\nabla\left(\tilde{M}_{\epsilon} u+\tilde{N}_{\epsilon} u\right)\right|^{2} d x \\
& =\int_{\Omega_{\epsilon}}\left|\nabla\left(\tilde{M}_{\epsilon} u+\tilde{N}_{\epsilon} u\right) \cdot \nabla\left(\tilde{M}_{\epsilon} u+\tilde{N}_{\epsilon} u\right)\right| d x \\
& =\left\|\tilde{M}_{\epsilon} u\right\|_{\epsilon}^{2}+\left\|\tilde{N}_{\epsilon} u\right\|_{\epsilon}^{2}+ \\
& +\underbrace{2 \int_{\Omega_{\epsilon}}\left(\nabla \tilde{M}_{\epsilon} u \cdot \nabla \tilde{N}_{\epsilon} u\right) d x}_{=0 \text { por }(10)} .
\end{aligned}
$$

Logo,

$$
\|u\|_{\epsilon}^{2}=\left\|\tilde{M}_{\epsilon} u\right\|_{\epsilon}^{2}+\left\|\tilde{N}_{\epsilon} u\right\|_{\epsilon}^{2}
$$

(iii) Seja $v \in V_{\epsilon}$. Então, para qualquer condição de fronteira considerada, $v \in \mathbb{H}^{1}\left(\Omega_{\epsilon}\right)$. Pelo item (iv) da proposição anterior, conclui-se que $\tilde{M}_{\epsilon} v \in \mathbb{H}^{1}(\omega)$. Mas,

$$
\left\|\tilde{M}_{\epsilon} v\right\|_{\mathbb{L}^{2}\left(\Omega_{\epsilon}\right)}^{2}=\int_{\Omega_{\epsilon}}\left|\tilde{M}_{\epsilon} v\left(x_{1}, x_{2}, x_{3}\right)\right|^{2} d x=
$$




$$
\begin{gathered}
\int_{0}^{\epsilon} \int_{\omega}\left|\tilde{M}_{\epsilon} v\left(x_{1}, x_{2}, x_{3}\right)\right|^{2} d x_{1} d x_{2} d x_{3} .= \\
\int_{0}^{\epsilon}\left\|\tilde{M}_{\epsilon} v\right\|_{\mathbb{L}^{2}(\omega)}^{2} d x_{3}= \\
\left\|\tilde{M}_{\epsilon} v\right\|_{\mathbb{L}^{2}(\omega)}^{2} \int_{0}^{\epsilon} d x_{3}= \\
\epsilon\left\|\tilde{M}_{\epsilon} v\right\|_{\mathbb{L}^{2}(\omega)}^{2} .
\end{gathered}
$$

Logo, como $\tilde{M}_{\epsilon} v \in \mathbb{H}^{1}(\omega)$, tem-se que $\left\|\tilde{M}_{\epsilon} v\right\|_{\mathbb{L}^{2}(\omega)}^{2}$ é finita e portanto $\tilde{M}_{\epsilon} v \in \mathbb{L}^{2}\left(\Omega_{\epsilon}\right)$. Analogamente, para $\frac{\partial}{\partial x_{i}} \tilde{M}_{\epsilon} v, i=1,2,3$, sendo que $\frac{\partial}{\partial x_{3}} \tilde{M}_{\epsilon} v \equiv 0$. Além disso, pelo item (v) da proposição anterior, $\tilde{M}_{\epsilon} v$ satisfaz a mesma condição de fronteira de $v$.

Para concluir que $\tilde{M}_{\epsilon} v \in V_{\epsilon}$, resta verificar se $\operatorname{div} \tilde{M}_{\epsilon} v=$ 0 . De fato, para as condições (DD) e (DP), como $\tilde{M}_{\epsilon} v=0$, segue que $\operatorname{div} \tilde{M}_{\epsilon} v=0$. Para as demais condições, temse que $\operatorname{div} v=0$, isto é,

$$
\operatorname{div} v=\frac{\partial v_{1}}{\partial x_{1}}+\frac{\partial v_{2}}{\partial x_{2}}+\frac{\partial v_{3}}{\partial x_{3}}=0
$$

Assim,

$$
\begin{array}{r}
0=\frac{1}{\epsilon} \int_{0}^{\epsilon} \operatorname{div} v d x_{3}= \\
\frac{1}{\epsilon}\left(\int_{0}^{\epsilon} \frac{\partial v_{1}}{\partial x_{1}} d x_{3}+\int_{0}^{\epsilon} \frac{\partial v_{2}}{\partial x_{2}} d x_{3}+\int_{0}^{\epsilon} \frac{\partial v_{3}}{\partial x_{3}} d x_{3}\right)= \\
\frac{\partial}{\partial x_{1}} \frac{1}{\epsilon} \int_{0}^{\epsilon} v_{1} d x_{3}+\frac{\partial}{\partial x_{2}} \frac{1}{\epsilon} \int_{0}^{\epsilon} v_{2} d x_{3}+\int_{0}^{\epsilon} \frac{\partial v_{3}}{\partial x_{3}} d x_{3},
\end{array}
$$

isto é,

$$
\frac{\partial}{\partial x_{1}} M_{\epsilon} v_{1}+\frac{\partial}{\partial x_{2}} M_{\epsilon} v_{2}=-\int_{0}^{\epsilon} \frac{\partial v_{3}}{\partial x_{3}} d x_{3}
$$

Mas, pelo Teorema Fundamental do Cálculo,

$$
\int_{0}^{\epsilon} \frac{\partial v_{3}}{\partial x_{3}} d x_{3}=\frac{1}{\epsilon} v_{3}\left(x_{1}, x_{2}, \epsilon\right)-\frac{1}{\epsilon} v_{3}\left(x_{1}, x_{2}, 0\right)=0
$$

pois, para (FP), $v_{3}\left(x_{1}, x_{2}, \epsilon\right)=v_{3}\left(x_{1}, x_{2}, 0\right)=0$, para (PP), tomando $\epsilon$ como período, $v_{3}\left(x_{1}, x_{2}, \epsilon\right)=v_{3}\left(x_{1}, x_{2}, 0\right)$ e, para (FF), como $\tilde{M}_{\epsilon} v=\left(M_{\epsilon} v_{1}, M_{\epsilon} v_{2}, 0\right)$, tem-se que $M_{\epsilon} v_{3}=0$, ou seja, $\frac{1}{\epsilon} \int_{0}^{\epsilon} v_{3} d x_{3}=0$. Isto implica que

$$
0=\frac{\partial}{\partial x_{3}} \frac{1}{\epsilon} \int_{0}^{\epsilon} v_{3} d x_{3}=\frac{1}{\epsilon} \int_{0}^{\epsilon} \frac{\partial v_{3}}{\partial x_{3}} d x_{3}
$$

Portanto, de (11),

$$
\frac{\partial}{\partial x_{1}} M_{\epsilon} v_{1}+\frac{\partial}{\partial x_{2}} M_{\epsilon} v_{2}=0
$$

e, já que $\frac{\partial}{\partial x_{3}} M_{\epsilon} v_{3}=0$ pois $M_{\epsilon} v_{3}$ não depende de $x_{3}$,

$$
\operatorname{div} \tilde{M}_{\epsilon} v=0
$$

isto é, $\tilde{M}_{\epsilon} v \in V_{\epsilon}$. Além disso, $\tilde{N}_{\epsilon} v \in V_{\epsilon}$ pois $\tilde{N}_{\epsilon} v=$ $v-\tilde{M}_{\epsilon} v$ e $v, \tilde{M}_{\epsilon} v \in V_{\epsilon}$.

(iv) Sejam $u, v \in V_{\epsilon}$. Tem-se que

$$
\begin{aligned}
b_{\epsilon}\left(u, u, \tilde{M}_{\epsilon} v\right) & =b_{\epsilon}\left(\tilde{M}_{\epsilon} u+\tilde{N}_{\epsilon} u, \tilde{M}_{\epsilon} u+\tilde{N}_{\epsilon} u, \tilde{M}_{\epsilon} v\right) \\
& =b_{\epsilon}\left(\tilde{M}_{\epsilon} u, \tilde{M}_{\epsilon} u, \tilde{M}_{\epsilon} v\right)+b_{\epsilon}\left(\tilde{M}_{\epsilon} u, \tilde{N}_{\epsilon} u, \tilde{M}_{\epsilon} v\right) \\
& +b_{\epsilon}\left(\tilde{N}_{\epsilon} u, \tilde{M}_{\epsilon} u, \tilde{M}_{\epsilon} v\right)+b_{\epsilon}\left(\tilde{N}_{\epsilon} u, \tilde{N}_{\epsilon} u, \tilde{M}_{\epsilon} v\right) .
\end{aligned}
$$

Note que $b_{\epsilon}\left(\tilde{N}_{\epsilon} u, \tilde{M}_{\epsilon} u, \tilde{M}_{\epsilon} v\right)=0$, pois

$$
\begin{array}{r}
b_{\epsilon}\left(\tilde{N}_{\epsilon} u, \tilde{M}_{\epsilon} u, \tilde{M}_{\epsilon} v\right)=\sum_{i, j=1}^{3} \int_{\Omega_{\epsilon}} N_{\epsilon} u_{i} \frac{\partial M_{\epsilon} u_{j}}{\partial x_{i}} M_{\epsilon} v_{j} d x= \\
\sum_{i, j=1}^{3} \int_{\omega} \int_{0}^{\epsilon} N_{\epsilon} u_{i} \frac{\partial M_{\epsilon} u_{j}}{\partial x_{i}} M_{\epsilon} v_{j} d x= \\
\sum_{i, j=1}^{3} \int_{\omega}\left(\int_{0}^{\epsilon} N_{\epsilon} u_{i} d x_{3}\right) \frac{\partial M_{\epsilon} u_{j}}{\partial x_{i}} M_{\epsilon} v_{j} d x_{1} d x_{2} .
\end{array}
$$

Mas, pela propriedade (ii) da proposição anterior,

$$
\begin{aligned}
\int_{0}^{\epsilon} N_{\epsilon} u_{i} d x_{3}=\epsilon \frac{1}{\epsilon} \int_{0}^{\epsilon} N_{\epsilon} u_{i} d x_{3} & =\epsilon M_{\epsilon}\left(N_{\epsilon} u_{i}\right) \\
& =\epsilon\left(M_{\epsilon} \circ N_{\epsilon}\right)\left(u_{i}\right) \\
& =0,
\end{aligned}
$$

ou seja, a integral em (12) é nula. Além disso,

$$
b_{\epsilon}\left(\tilde{M}_{\epsilon} u, \tilde{N}_{\epsilon} u, \tilde{M}_{\epsilon} v\right)=0
$$

e, portanto,

$b_{\epsilon}\left(u, u, \tilde{M}_{\epsilon} v\right)=b_{\epsilon}\left(\tilde{M}_{\epsilon} u, \tilde{M}_{\epsilon} u, \tilde{M}_{\epsilon} v\right)+b_{\epsilon}\left(\tilde{N}_{\epsilon} u, \tilde{N}_{\epsilon} u, \tilde{M}_{\epsilon} v\right)$.

Analogamente, vale que

$$
\begin{aligned}
b_{\epsilon}\left(u, u, \tilde{N}_{\epsilon} v\right) & =b_{\epsilon}\left(\tilde{M}_{\epsilon} u, \tilde{M}_{\epsilon} u, \tilde{N}_{\epsilon} v\right)+b_{\epsilon}\left(\tilde{N}_{\epsilon} u, \tilde{M}_{\epsilon} u, \tilde{N}_{\epsilon} v\right) \\
& +b_{\epsilon}\left(\tilde{M}_{\epsilon} u, \tilde{N}_{\epsilon} u, \tilde{N}_{\epsilon} v\right)+b_{\epsilon}\left(\tilde{N}_{\epsilon} u, \tilde{N}_{\epsilon} u, \tilde{N}_{\epsilon} v\right) .
\end{aligned}
$$

$\operatorname{Mas} b_{\epsilon}\left(\tilde{M}_{\epsilon} u, \tilde{M}_{\epsilon} u, \tilde{N}_{\epsilon} v\right)=0$, já que

$$
\begin{gathered}
b_{\epsilon}\left(\tilde{M}_{\epsilon} u, \tilde{M}_{\epsilon} u, \tilde{N}_{\epsilon} v\right)=\sum_{i, j=1}^{3} \int_{\Omega_{\epsilon}} M_{\epsilon} u_{i} \frac{\partial M_{\epsilon} u_{j}}{\partial x_{i}} N_{\epsilon} v_{j} d x= \\
\sum_{i, j=1}^{3} \int_{\omega} \underbrace{\left(\int_{0}^{\epsilon} N_{\epsilon} v_{j} d x_{3}\right)}_{=0} M_{\epsilon} u_{i} \frac{\partial M_{\epsilon} u_{j}}{\partial x_{i}} d x_{1} d x_{2}=0 .
\end{gathered}
$$

Logo,

$$
\begin{aligned}
b_{\epsilon}\left(u, u, \tilde{N}_{\epsilon} v\right) & =b_{\epsilon}\left(\tilde{N}_{\epsilon} u, \tilde{M}_{\epsilon} u, \tilde{N}_{\epsilon} v\right)+b_{\epsilon}\left(\tilde{M}_{\epsilon} u, \tilde{N}_{\epsilon} u, \tilde{N}_{\epsilon} v\right) \\
& +b_{\epsilon}\left(\tilde{N}_{\epsilon} u, \tilde{N}_{\epsilon} u, \tilde{N}_{\epsilon} v\right) .
\end{aligned}
$$

(v) Para as condições de fronteira (DP) ou (DD), temse $\tilde{M}_{\epsilon} u=0$, para $u \in D\left(A_{\epsilon}\right)$. Neste caso,

$$
\Delta \tilde{M}_{\epsilon} u=0 \text {, }
$$


e

$$
\tilde{M}_{\epsilon} \Delta u=\tilde{M}_{\epsilon}\left(\Delta u_{1}, \Delta u_{2}, \Delta u_{3}\right)=0,
$$

visto que, como $u \in D\left(A_{\epsilon}\right)$, então $u \in \mathbb{H}^{2}\left(\Omega_{\epsilon}\right)$ e assim $\Delta u \in \mathbb{L}^{2}\left(\Omega_{\epsilon}\right)$. Portanto, $\Delta \tilde{M}_{\epsilon} u=\tilde{M}_{\epsilon} \Delta u$. Ainda, tem-se que $\tilde{N}_{\epsilon} u=u-\tilde{M}_{\epsilon} u=u-0=u$, isto é, $\Delta \tilde{N}_{\epsilon} u=\Delta u$. Por outro lado,

$$
\tilde{N}_{\epsilon} \Delta u=\Delta u-\tilde{M}_{\epsilon} \Delta u=\Delta u-0=\Delta u .
$$

Logo, $\Delta \tilde{N}_{\epsilon} u=\tilde{N}_{\epsilon} \Delta u$.

Para as condições de fronteira (FP), (FF), sejam $v \in$ $C_{0}^{\infty}(\omega) \times C_{0}^{\infty}(\omega)$ e $u \in D\left(A_{\epsilon}\right)$. Como

$$
\tilde{M}_{\epsilon} u=\left(M_{\epsilon} u_{1}, M_{\epsilon} u_{2}, 0\right),
$$

tem-se

$$
\begin{array}{r}
\int_{\omega} \Delta\left(\tilde{M}_{\epsilon} u\right) \cdot v d x_{1} d x_{2}= \\
\int_{\omega}\left(\Delta^{\prime} M_{\epsilon} u_{1}, \Delta^{\prime} M_{\epsilon} u_{2}, 0\right) \cdot\left(v_{1}, v_{2}, 0\right) d x_{1} d x_{2}= \\
\sum_{i=1}^{2} \int_{\omega} \Delta^{\prime} M_{\epsilon} u_{i} v_{i} d x_{1} d x_{2} .
\end{array}
$$

Usando a fórmula de Green, então

$$
\begin{array}{r}
\sum_{i=1}^{2} \int_{\omega} \Delta^{\prime} M_{\epsilon} u_{i} v_{i} d x_{1} d x_{2}= \\
-\sum_{i=1}^{2} \int_{\omega} \nabla^{\prime}\left(M_{\epsilon} u_{i}\right) \cdot \nabla^{\prime} v_{i} d x_{1} d x_{2} \\
\sum_{i=1}^{2} \int_{\partial \omega} v_{i} \frac{\partial}{\partial \mathbf{n}} M_{\epsilon} u_{i} d S= \\
-\sum_{i=1}^{2} \int_{\omega} \nabla^{\prime}\left(M_{\epsilon} u_{i}\right) \cdot \nabla^{\prime} v_{i} d x_{1} d x_{2},
\end{array}
$$

pois $v \equiv 0$ em $\partial \omega$ e $\mathbf{n}$ é a normal de $\partial \omega$. Assim, pela propriedade (iii) da proposição anterior,

$$
\begin{array}{r}
-\sum_{i=1}^{2} \int_{\omega} \nabla^{\prime}\left(M_{\epsilon} u_{i}\right) \cdot \nabla^{\prime} v_{i} d x_{1} d x_{2}= \\
-\sum_{i=1}^{2} \int_{\omega} \tilde{M}_{\epsilon}\left(\nabla^{\prime} u_{i}\right) \cdot \nabla^{\prime} v_{i} d x_{1} d x_{2}= \\
-\sum_{i=1}^{2} \int_{\omega}\left(\frac{1}{\epsilon} \int_{0}^{\epsilon}\left(\nabla^{\prime} u_{i}\right) d x_{3}\right) \cdot \nabla^{\prime} v_{i} d x_{1} d x_{2}= \\
-\frac{1}{\epsilon} \sum_{i=1}^{2} \int_{\Omega_{\epsilon}} \nabla u_{i} \cdot \nabla v_{i} d x .
\end{array}
$$

Novamente, pela fórmula de Green,

$$
-\frac{1}{\epsilon} \sum_{i=1}^{2} \int_{\Omega_{\epsilon}} \nabla u_{i} \cdot \nabla v_{i} d x=
$$

$$
\frac{1}{\epsilon} \sum_{i=1}^{2} \int_{\Omega_{\epsilon}} v_{i} \Delta u_{i} d x-\frac{1}{\epsilon} \sum_{i=1}^{2} \int_{\partial \Omega_{\epsilon}} v_{i} \frac{\partial u_{i}}{\partial \mathbf{n}} d S .
$$

Note que

$$
\begin{aligned}
\int_{\partial \Omega_{\varepsilon}} v_{i} \frac{\partial u_{i}}{\partial \mathbf{n}} d S & =\int_{\Gamma_{t}} v_{i} \frac{\partial u_{i}}{\partial \mathbf{n}} d S+\int_{\Gamma_{l}} v_{i} \frac{\partial u_{i}}{\partial \mathbf{n}} d S \\
& +\int_{\Gamma_{b}} v_{i} \frac{\partial u_{i}}{\partial \mathbf{n}} d S .
\end{aligned}
$$

A segunda integral depois da igualdade de (15) é nula, pois se $x \in \Gamma_{l}$, então $\left(x_{1}, x_{2}\right) \in \partial \omega$ e $v_{i}\left(x_{1}, x_{2}\right)=0$, já que $v_{i} \in C_{0}^{\infty}(\omega)$. Agora, quando $x \in \Gamma_{t}$ ou $x \in \Gamma_{b}$, tem-se que $\mathbf{n}= \pm e_{3}=(0,0,1)$ e assim

$$
\frac{\partial u_{i}}{\partial \mathbf{n}}=\frac{\partial u_{i}}{\partial x_{3}} \quad \text { ou } \quad \frac{\partial u_{i}}{\partial \mathbf{n}}=-\frac{\partial u_{i}}{\partial x_{3}}
$$

e, de acordo com a condição (FP), deve-se ter $\frac{\partial u_{i}}{\partial x_{3}}=0$ para $i=1,2$.

Já para a condição de fronteira (FF), observa-se que

$$
\operatorname{rot} u=\left(\frac{\partial u_{3}}{\partial x_{2}}-\frac{\partial u_{2}}{\partial x_{3}}, \frac{\partial u_{1}}{\partial x_{3}}-\frac{\partial u_{3}}{\partial x_{2}}, \frac{\partial u_{2}}{\partial x_{1}}-\frac{\partial u_{1}}{\partial x_{2}}\right)
$$

e $\mathbf{n}=(0,0,1)$ em $\Gamma_{t}$ e $\Gamma_{b}, \log$,

$$
\operatorname{rot} u \times \mathbf{n}=0 \Rightarrow\left(\frac{\partial u_{1}}{\partial x_{3}}-\frac{\partial u_{3}}{\partial x_{2}}, \frac{\partial u_{2}}{\partial x_{3}}-\frac{\partial u_{3}}{\partial x_{2}}, 0\right)=0,
$$

ou seja,

$$
\frac{\partial u_{1}}{\partial x_{3}}-\frac{\partial u_{3}}{\partial x_{2}}=0 \quad \text { e } \quad \frac{\partial u_{2}}{\partial x_{3}}-\frac{\partial u_{3}}{\partial x_{2}}=0 .
$$

Mas $u \cdot \mathbf{n}=0$ em $\Gamma_{t}$ e $\Gamma_{b}$, isto é, $u_{3}=0$ e $\frac{\partial u_{3}}{\partial x_{2}}=0$. Assim,

$$
\frac{\partial u_{1}}{\partial x_{3}}=\frac{\partial u_{2}}{\partial x_{3}}=0 .
$$

Como o somatório em (13) só compreende $i=1,2$, segue que a primeira e a terceira integral depois da igualdade de (15) são nulas e portanto

$$
\int_{\partial \Omega_{\varepsilon}} v_{i} \frac{\partial u_{i}}{\partial \mathbf{n}} d S=0
$$

Substituindo em (14), tem-se

$$
\begin{array}{r}
-\frac{1}{\epsilon} \sum_{i=1}^{2} \int_{\Omega_{\epsilon}} \nabla u_{i} \cdot \nabla v_{i} d x=\frac{1}{\epsilon} \sum_{i=1}^{2} \int_{\Omega_{\epsilon}} v_{i} \Delta u_{i} d x= \\
\frac{1}{\epsilon} \sum_{i=1}^{2} \int_{\omega} \int_{0}^{\epsilon} v_{i} \Delta u_{i} d x=\frac{1}{\epsilon} \sum_{i=1}^{2} \int_{\omega} v_{i}\left(\int_{0}^{\epsilon} \Delta u_{i}\right) d x= \\
\left.\int_{\omega}^{2} \int_{i=1} \int_{\omega} v_{i} M_{\epsilon}, v_{2}\right) \cdot \underbrace{\left(M_{\epsilon} \Delta u_{1}, M_{\epsilon} \Delta u_{2}\right)}_{\tilde{M}_{\epsilon}\left(\Delta^{\prime} u\right)} d x_{2}=
\end{array}
$$




$$
=\int_{\omega} \tilde{M}_{\epsilon}(\Delta u) \cdot v d x_{1} d x_{2}
$$

Conclui-se que

$$
\int_{\omega} \Delta\left(\tilde{M}_{\epsilon} u\right) \cdot v d x_{1} d x_{2}=\int_{\omega} \tilde{M}_{\epsilon}(\Delta u) \cdot v d x_{1} d x_{2}
$$

ou seja,

$$
\int_{\omega}\left(\tilde{M}_{\epsilon}(\Delta u)-\Delta\left(\tilde{M}_{\epsilon} u\right)\right) \cdot v d x_{1} d x_{2}=0
$$

Assim, $\tilde{M}_{\epsilon}(\Delta u)=\Delta\left(\tilde{M}_{\epsilon} u\right)$ q.s. em $\omega$. Além disso,

$$
\Delta u=\Delta \tilde{M}_{\epsilon} u+\Delta \tilde{N}_{\epsilon} u=\tilde{M}_{\epsilon}(\Delta u)+\Delta \tilde{N}_{\epsilon} u .
$$

Mas por outro lado,

$$
\tilde{N}_{\epsilon}(\Delta u)=\Delta u-\tilde{M}_{\epsilon}(\Delta u) \Rightarrow \Delta u=\tilde{M}_{\epsilon}(\Delta u)+\tilde{N}_{\epsilon}(\Delta u) .
$$

Portanto, $\tilde{N}_{\epsilon}(\Delta u)=\Delta \tilde{N}_{\epsilon} u$.

Além destes resultados, os operadores $\tilde{M}_{\epsilon}$ e $\tilde{N}_{\epsilon}$ também comutam-se com o operador de Stokes $A_{\epsilon}$, isto é,

$$
A_{\epsilon} \tilde{M}_{\epsilon} u=\tilde{M}_{\epsilon} A_{\epsilon} u \quad \text { e } \quad A_{\epsilon} \tilde{N}_{\epsilon} u=\tilde{N}_{\epsilon} A_{\epsilon} u
$$

onde $u \in D\left(A_{\epsilon}\right)$. Para as condições (FF) e (FP), ainda temos o seguinte resultado:

Lema 2.2. Se $u \in D\left(A_{\epsilon}\right)$ satisfaz $(F F)$ e $(F P)$, então

$$
b_{\epsilon}\left(\tilde{M}_{\epsilon} u, \tilde{M}_{\epsilon} u, A_{\epsilon}\left(\tilde{M}_{\epsilon} u,\right)\right)=0
$$

\subsection{Desigualdades Fundamentais em Domí- nios Finos}

Nesta seção, serão enunciados resultados para desigualdades clássicas em domínios finos. Tal ferramenta torna-se importante para conhecer a exata dependência das constantes que aparecem em desigualdades do tipo Sobolev com relação à espessura do domínio. Algumas serão apenas enunciadas, pelo fato de suas demonstrações serem extensas e fugirem do escopo principal deste trabalho.

A primeira desigualdade aqui enunciada é a de Poincaré, cuja demonstração pode ser encontrada em Temam e Ziane (1996).

Proposição 2.2 (Desigualdade de Poincaré). Seja $u \in$ $H^{1}\left(\Omega_{\epsilon}\right)$ satisfazendo:

$$
\left\{\begin{array}{l}
u \equiv 0 \text { em } \Gamma_{t} \cup \Gamma_{b} \\
\int_{0}^{\epsilon} u\left(x_{1}, x_{2}, x_{3}\right) d x_{3}=0 \text { q.s. em } \omega .
\end{array}\right.
$$

Então,

$$
|u|_{\epsilon} \leq \epsilon\left|\frac{\partial u}{\partial x_{3}}\right|_{\epsilon}
$$

Vale notar que a desigualdade acima vale para qualquer condição de fronteira a ser considerada. Como visto no lema (2.1), se $u \in V_{\epsilon}$ então $\tilde{N}_{\epsilon} u \in V_{\epsilon}$ e portanto, tem-se o seguinte resultado.

Corolário 2.1. Para qualquer $u \in V_{\epsilon}$, vale que

$$
\left|\tilde{N}_{\epsilon} u\right|_{\epsilon} \leq \epsilon\left|\frac{\partial \tilde{N}_{\epsilon} u}{\partial x_{3}}\right|_{\epsilon}
$$

Corolário 2.2. Sob as mesmas hipóteses da proposição 2.2, tem-se

$$
|u|_{\epsilon} \leq \epsilon|\nabla u|_{\epsilon}
$$

Demonstração: De fato, basta notar que

$$
\begin{aligned}
|u|_{\epsilon} & \leq \epsilon\left|\frac{\partial u}{\partial x_{3}}\right|_{\epsilon}=\epsilon \sqrt{\left|\frac{\partial u}{\partial x_{3}}\right|_{\epsilon}^{2}} \\
& \leq \epsilon \sqrt{\left|\frac{\partial u}{\partial x_{1}}\right|_{\epsilon}^{2}+\left|\frac{\partial u}{\partial x_{2}}\right|_{\epsilon}^{2}+\left|\frac{\partial u}{\partial x_{3}}\right|_{\epsilon}^{2}} \\
& =\epsilon|\nabla u|_{\epsilon} .
\end{aligned}
$$

Corolário 2.3. Sob as mesmas hipóteses da proposição 2.2, tem-se

$$
\|u\|_{\epsilon} \leq \epsilon\left|A_{\epsilon} u\right|_{\epsilon}
$$

Demonstração: Da definição do operador de Stokes, $A_{\epsilon}$ é definido positivo, isto é, vale

$$
\left(A_{\epsilon} u, u\right)_{\epsilon}=\|u\|_{\epsilon}^{2}>0 .
$$

Mas, pela desigualdade de Holder, tomando $p=q=2$, tem-se

$$
\left(A_{\epsilon} u, u\right)_{\epsilon} \leq\left|A_{\epsilon} u\right|_{\epsilon}|u|_{\epsilon}
$$

Logo,

$$
\|u\|_{\epsilon}^{2} \leq\left|A_{\epsilon} u\right|_{\epsilon}|u|_{\epsilon}
$$

Usando o corolário anterior,

$$
\|u\|_{\epsilon}^{2} \leq\left|A_{\epsilon} u\right|_{\epsilon} \epsilon|\nabla u|_{\epsilon}=\epsilon\left|A_{\epsilon} u\right|_{\epsilon}\|u\|_{\epsilon}
$$

Portanto,

$$
\|u\|_{\epsilon} \leq \epsilon\left|A_{\epsilon} u\right|_{\epsilon}
$$

Uma outra desigualdade importante é a de Agmon, a qual será apresentada aqui na sua forma específica para domínios finos. A demonstração pode ser encontrada em Temam e Ziane (1996). 
Corolário 2.4 (Desigualdade Anisotrópica de Agmon para Domínios Finos). Existe uma constante positiva $c_{0}(\omega)$, que não depende de $\epsilon$, tal que, para toda $u \in H^{2}\left(\Omega_{\epsilon}\right)$,

$$
\begin{aligned}
|u|_{L^{\infty}\left(\Omega_{\epsilon}\right)} & \leq c_{0}(\omega)|u|_{\epsilon}^{1 / 4} \cdot\left(\left|\frac{\partial^{2} u}{\partial x_{3}^{2}}\right|_{\epsilon}+\frac{1}{\epsilon}\left|\frac{\partial u}{\partial x_{3}}\right|_{L^{2}\left(\Omega_{\epsilon}\right)}\right. \\
& \left.+\frac{1}{\epsilon^{2}}|u|_{L^{2}\left(\Omega_{\epsilon}\right)}\right)^{1 / 4} \cdot \prod_{i=1}^{2}\left(\sum_{j=1}^{2}\left|\frac{\partial^{2} u}{\partial x_{i} \partial x_{j}}\right|_{\epsilon}\right. \\
& \left.+\left|\frac{\partial u}{\partial x_{j}}\right|_{\epsilon}+|u|_{\epsilon}\right)^{1 / 4} .
\end{aligned}
$$

Corolário 2.5. Suponha que $u \in D\left(A_{\epsilon}\right)$. Então, para qualquer condição de fronteira, vale

$$
\left|\frac{\partial N_{\epsilon} u_{j}}{\partial x_{i}}\right|_{\epsilon} \leq \epsilon\left|\frac{\partial^{2} N_{\epsilon} u_{j}}{\partial x_{i} \partial x_{3}}\right|_{\epsilon}, \text { para } i, j=1,2,3 \text {. }
$$

Demonstração: Observe que, para $i=1,2$, devido à (2), tem-se

$$
\frac{\partial N_{\epsilon} u_{j}}{\partial x_{i}}=0 \text { em } \Gamma_{t} \cup \Gamma_{b} \quad \text { e } \int_{0}^{\epsilon} \frac{\partial N_{\epsilon} u_{j}}{\partial x_{i}} d x_{3}=0,
$$

com $j=1,2,3$. Sendo assim, $\frac{\partial N_{\epsilon} u_{j}}{\partial x_{i}}$ satisfaz (16). Logo, pela Desigualdade de Poincaré,

$$
\left|\frac{\partial N_{\epsilon} u_{j}}{\partial x_{i}}\right|_{\epsilon} \leq \epsilon\left|\frac{\partial^{2} N_{\epsilon} u_{j}}{\partial x_{i} \partial x_{3}}\right|_{\epsilon} .
$$

Agora, para a dimensão espacial $x_{3}$, deve-se ter um pouco mais de cuidado, visto que não é possível passar a derivada com relação à $x_{3}$ para dentro da integral em (2). Portanto, usando integração por partes em $x_{3}$, temos

$$
\int_{\Omega_{\epsilon}} N_{\epsilon} u_{j} \frac{\partial^{2} N_{\epsilon} u_{j}}{\partial x_{3}^{2}} d x=-\int_{\Omega_{\epsilon}}\left(\frac{\partial N_{\epsilon} u_{j}}{\partial x_{3}}\right)^{2} d x .
$$

Assim,

$$
\begin{aligned}
\int_{\Omega_{\epsilon}}\left(\frac{\partial N_{\epsilon} u_{j}}{\partial x_{3}}\right)^{2} d x & =-\int_{\Omega_{\epsilon}} N_{\epsilon} u_{j} \frac{\partial^{2} N_{\epsilon} u_{j}}{\partial x_{3}^{2}} d x \\
& \leq \int_{\Omega_{\epsilon}}\left|N_{\epsilon} u_{j}\right| \frac{\partial^{2} N_{\epsilon} u_{j}}{\partial x_{3}^{2}} \mid d x \\
& \leq\left|N_{\epsilon} u_{j}\right|_{L^{2}\left(\Omega_{\epsilon}\right)}\left|\frac{\partial^{2} N_{\epsilon} u_{j}}{\partial x_{3}^{2}}\right|_{L^{2}\left(\Omega_{\epsilon}\right)} \\
\left|\frac{\partial N_{\epsilon} u_{j}}{\partial x_{3}}\right|_{\epsilon}^{2} & \leq\left.\left|N_{\epsilon} u_{j}\right|_{\epsilon}\left|\frac{\partial^{2} N_{\epsilon} u_{j}}{\partial x_{3}^{2}}\right|_{\epsilon}\right|_{\text {pelo Corol. (2.1) }} \leq \epsilon\left|\frac{\partial N_{\epsilon} u_{j}}{\partial x_{3}}\right|_{\epsilon}\left|\frac{\partial^{2} N_{\epsilon} u_{j}}{\partial x_{3}^{2}}\right|_{\epsilon}
\end{aligned}
$$

ou seja,

$$
\left|\frac{\partial N_{\epsilon} u_{j}}{\partial x_{3}}\right|_{\epsilon} \leq\left|\frac{\partial^{2} N_{\epsilon} u_{j}}{\partial x_{3}^{2}}\right|_{\epsilon}
$$

Combinando os corolários 2.1 e 2.4, resulta o que segue.

Corolário 2.6. Existe uma constante positiva $c_{0}(\omega)$, que não depende de $\epsilon$, tal que, para toda $u \in D\left(A_{\epsilon}\right)$,

$$
\left|\tilde{N}_{\epsilon} u\right|_{L^{\infty}\left(\Omega_{\epsilon}\right)} \leq c_{0}(\omega)\left|\tilde{N}_{\epsilon} u\right|_{\epsilon}^{1 / 4}\left(\sum_{i, j=1}^{3}\left|\frac{\partial \tilde{N}_{\epsilon} u}{\partial x_{i} \partial x_{j}}\right|_{\epsilon}\right)^{3 / 4}
$$

Corolário 2.7. Existe uma constante positiva $c_{0}$, independente de $\epsilon$, tal que

$$
\left|\tilde{N}_{\epsilon} u\right|_{L^{6}\left(\Omega_{\epsilon}\right)}^{2} \leq c_{0}\left\|\tilde{N}_{\epsilon} u\right\|_{\epsilon}^{2} .
$$

A partir do corolário acima e da proposição 2.2, obtem-se o seguinte lema.

Lema 2.3. Para $2 \leq q \leq 6$, existe uma constante positiva $c(q)$, independente de $\epsilon$ tal que, para todo $u \in V_{\epsilon}$,

$$
\left|\tilde{N}_{\epsilon} u\right|_{L^{q}\left(\Omega_{\epsilon}\right)}^{2} \leq c(q) \epsilon^{\frac{6-q}{q}}\left\|\tilde{N}_{\epsilon} u\right\|_{\epsilon}^{2}
$$

Teorema 2.1. Sob qualquer uma das condições de fronteira consideradas, existe uma constante $c$, independente de $\epsilon$, tal que, para todo $u \in D\left(A_{\epsilon}\right)$,

$$
\sum_{i, j=1}^{3}\left|\frac{\partial^{2} u}{\partial x_{i} \partial x_{j}}\right|_{\epsilon}^{2} \leq c\left|A_{\epsilon} u\right|_{\epsilon}^{2} .
$$

Por fim, o próximo resultado está relacionado com desigualdades para a forma trilinear $b_{\epsilon}$.

Lema 2.4. Seja $0<q<\frac{1}{2}$. Existe uma constante positiva $c_{4}(q)$, que não depende de $\epsilon$, tal que, para qualquer condição de fronteira considerada, tem-se

(i) $\left|b_{\epsilon}\left(\tilde{M}_{\epsilon} u, \tilde{N}_{\epsilon} u, w\right)\right| \leq\left. c_{4} \epsilon^{q}|| \tilde{M}_{\epsilon} u\right|_{\epsilon}\left|A_{\epsilon} \tilde{N}_{\epsilon} u\right|_{\epsilon}|w|_{\epsilon^{\prime}}$ $\forall u \in D\left(A_{\epsilon}\right) e w \in \mathbb{L}^{2}\left(\Omega_{\epsilon}\right)$.

(ii) $\left|b_{\epsilon}\left(\tilde{N}_{\epsilon} u, \tilde{M}_{\epsilon} u, w\right)\right| \leq c_{4} \epsilon^{1 / 2}|| \tilde{M}_{\epsilon} u||_{\epsilon}\left|A_{\epsilon} \tilde{N}_{\epsilon} u\right|_{\epsilon}|w|_{\epsilon^{\prime}}$ $\forall u \in D\left(A_{\epsilon}\right) e w \in \mathbb{L}^{2}\left(\Omega_{\epsilon}\right)$.

(iii) $\left|b_{\epsilon}\left(\tilde{N}_{\epsilon} u, \tilde{N}_{\epsilon} u, w\right)\right| \leq\left. c_{4}|| \tilde{N}_{\epsilon} u\right|_{\epsilon} ^{3 / 2}\left|A_{\epsilon} \tilde{N}_{\epsilon} u\right|_{\epsilon}^{1 / 2}|w|_{\epsilon}$ $\leq c_{4} \epsilon^{1 / 2}|| \tilde{N}_{\epsilon} u \|_{\epsilon}\left|A_{\epsilon} \tilde{N}_{\epsilon} u\right|_{\epsilon}|w|_{\epsilon^{\prime}}$ $\forall u \in D\left(A_{\epsilon}\right) e w \in \mathbb{L}^{2}\left(\Omega_{\epsilon}\right)$.

A demonstração do lema acima pode ser encontrado em Temam e Ziane (1996).

\section{Solução Fraca em Domínios Finos}

Com relação a existência de solução das equações de Navier-Stokes em domínios gerais, o seguinte resultado é conhecido em diversas bibliografias, tais como Temam (Temam (1984), Temam (1995)) e Lions (Lions (1969)). Ambos usam o método de Galerkin para encontrar solução fraca em espaços de Sobolev. Mais precisamente, tem-se 
Teorema 3.1. Sejam $u_{0} \in H$ e $f \in L^{2}(0, T, V)$. Então existe

$$
u \in L^{2}(0, T, V) \cap L^{\infty}(0, T, H)
$$

para todo $T>0$, solução das equações de Navier-Stokes. Se $u_{0} \in V$ então existe $T=T\left(\Omega, \mu, u_{0}, f\right)>0$ tal que

$$
u \in L^{2}\left(0, T, D\left(A_{\epsilon}\right)\right) \cap L^{\infty}(0, T, V)
$$

é a única solução das equações de Navier-Stokes.

No que segue, a solução única dada pelo teorema acima é chamada de solução forte.

\subsection{Estimativas para $\tilde{M}_{\epsilon}$ e $\tilde{N}_{\epsilon}$}

As desigualdades apresentadas na última seção motivam algumas estimativas a priori para os operadores $\tilde{M}_{\epsilon}$ e $\tilde{N}_{\epsilon}$, as quais formam o principal mecanismo para o estudo do tempo máximo de existência das soluções. Primeiramente, a partir das equações de Navier-Stokes, faz-se a formulação fraca para $\tilde{M}_{\epsilon}$ e $\tilde{N}_{\epsilon}$.

Antes de demonstrar a formulação fraca, observe que como $(u, v)_{D\left(A^{1 / 2}\right)}=\left(\left(A_{\epsilon}^{1 / 2} u, A_{\epsilon}^{1 / 2} v\right)\right)_{\epsilon}$, segue que

$$
\begin{aligned}
\left(\left(A_{\epsilon}^{1 / 2} \tilde{M}_{\epsilon} u, A_{\epsilon}^{1 / 2} \tilde{M}_{\epsilon} v\right)\right)_{\epsilon} & =\left(\tilde{M}_{\epsilon} u, \tilde{M}_{\epsilon} v\right)_{D\left(A_{\epsilon}^{1 / 2}\right)} \\
& =\left(\nabla \tilde{M}_{\epsilon} u, \nabla \tilde{M}_{\epsilon} v\right)_{\epsilon} .
\end{aligned}
$$

Note que faz sentido tomar o produto interno com relação a $\tilde{M}_{\epsilon} u$ e $\tilde{M}_{\epsilon} v$, pois, como $u, v \in D\left(A_{\epsilon}^{1 / 2}\right)=V_{\epsilon}$, tem-se pelo lema 2.1 que $\tilde{M}_{\epsilon} u, \tilde{M}_{\epsilon} v \in V_{\epsilon}$.

Proposição 3.1 (Formulação fraca para $\tilde{M}_{\epsilon}$ e $\tilde{N}_{\epsilon}$ ). Sejam $v \in V_{\epsilon}$ e $u \in H_{\epsilon}$. Então,

$$
\begin{gathered}
\text { (i) } \frac{d}{d t}\left(\tilde{M}_{\epsilon} u, \tilde{M}_{\epsilon} v\right)_{\epsilon}+\mu\left(A_{\epsilon}^{1 / 2} \tilde{M}_{\epsilon} u, A_{\epsilon}^{1 / 2} \tilde{M}_{\epsilon} v\right)_{\epsilon} \\
+b_{\epsilon}\left(\tilde{M}_{\epsilon} u, \tilde{M}_{\epsilon} u, \tilde{M}_{\epsilon} v\right) \\
+b_{\epsilon}\left(\tilde{N}_{\epsilon} u, \tilde{N}_{\epsilon} u, \tilde{M}_{\epsilon} v\right)=\left(\tilde{M}_{\epsilon} f, \tilde{M}_{\epsilon} v\right)_{\epsilon} \\
\text { (ii) } \frac{d}{d t}\left(\tilde{N}_{\epsilon} u, \tilde{N}_{\epsilon} v\right)_{\epsilon}+\mu\left(A_{\epsilon}^{1 / 2} \tilde{N}_{\epsilon} u, A_{\epsilon}^{1 / 2} \tilde{N}_{\epsilon} v\right)_{\epsilon} \\
+b_{\epsilon}\left(\tilde{M}_{\epsilon} u, \tilde{N}_{\epsilon} u, \tilde{N}_{\epsilon} v\right)+b_{\epsilon}\left(\tilde{N}_{\epsilon} u, \tilde{M}_{\epsilon} u, \tilde{N}_{\epsilon} v\right) \\
+b_{\epsilon}\left(\tilde{N}_{\epsilon} u, \tilde{N}_{\epsilon} u, \tilde{N}_{\epsilon} v\right)=\left(\tilde{N}_{\epsilon} f, \tilde{N}_{\epsilon} v\right)_{\epsilon}
\end{gathered}
$$

Demonstração: Considere a forma vetorial das equações de Navier-Stokes,

$$
u_{t}+u \cdot \nabla u-\mu \Delta u+\nabla p=f .
$$

Aplicando o operador $\tilde{M}_{\epsilon}$ em cada componente da equação acima, tem-se

$$
\tilde{M}_{\epsilon} u_{t}+\tilde{M}_{\epsilon}(u \cdot \nabla u)-\mu \tilde{M}_{\epsilon} \Delta u+\tilde{M}_{\epsilon} \nabla p=\tilde{M}_{\epsilon} f .
$$

Observe que $\tilde{M}_{\epsilon} u_{t}=\left(\tilde{M}_{\epsilon} u\right)_{t}$ e $\mu \tilde{M}_{\epsilon} \Delta u=\mu \Delta\left(\tilde{M}_{\epsilon} u\right)$. Ainda,

$$
\begin{aligned}
\tilde{M}_{\epsilon} \nabla p & =\tilde{M}_{\epsilon}\left(\frac{\partial p}{\partial x_{1}}, \frac{\partial p}{\partial x_{2}}, \frac{\partial p}{\partial x_{3}}\right) \\
& =\left(M_{\epsilon}\left(\frac{\partial p}{\partial x_{1}}\right), M_{\epsilon}\left(\frac{\partial p}{\partial x_{2}}\right), M_{\epsilon}\left(\frac{\partial p}{\partial x_{3}}\right)\right) \\
& =(\frac{\partial}{\partial x_{1}}\left(M_{\epsilon} p\right), \frac{\partial}{\partial x_{2}}\left(M_{\epsilon} p\right), \underbrace{\frac{\partial}{\partial x_{3}}\left(M_{\epsilon} p\right)}_{=0}) \\
& =\nabla^{\prime} \tilde{M}_{\epsilon} p .
\end{aligned}
$$

Multiplicando (18) por $\tilde{M}_{\epsilon} v$ e integrando em $\Omega_{\epsilon}$ tem-se

$$
\begin{aligned}
\left(\left(\tilde{M}_{\epsilon} u\right)_{t}, \tilde{M}_{\epsilon} v\right)_{\epsilon} & +\left(\tilde{M}_{\epsilon}(u \cdot \nabla u)_{,} \tilde{M}_{\epsilon} v\right)_{\epsilon} \\
& -\mu\left(\Delta \tilde{M}_{\epsilon} u, \tilde{M}_{\epsilon} v\right)_{\epsilon} \\
& +\left(\nabla^{\prime} \tilde{M}_{\epsilon} p, \tilde{M}_{\epsilon} v\right)_{\epsilon} \\
& =\left(\tilde{M}_{\epsilon} f, \tilde{M}_{\epsilon} v\right)_{\epsilon} .
\end{aligned}
$$

Agora, note que

$$
\begin{aligned}
\frac{d}{d t}\left(\tilde{M}_{\epsilon} u, \tilde{M}_{\epsilon} v\right)_{\epsilon} & =\left(\left(\tilde{M}_{\epsilon} u\right)_{t}, \tilde{M}_{\epsilon} v\right)_{\epsilon} \\
& +\left(\tilde{M}_{\epsilon} u,\left(\tilde{M}_{\epsilon} v\right)_{t}\right)_{\epsilon}
\end{aligned}
$$

e, como $v \in V_{\epsilon}$, não depende de $t$, donde $\left(\tilde{M}_{\epsilon} u,\left(\tilde{M}_{\epsilon} v\right)_{t}\right)=$ 0. Logo,

$$
\frac{d}{d t}\left(\tilde{M}_{\epsilon} u, \tilde{M}_{\epsilon} v\right)_{\epsilon}=\left(\left(\tilde{M}_{\epsilon} u\right)_{t}, \tilde{M}_{\epsilon} v\right)_{\epsilon} .
$$

Como $\tilde{M}_{\epsilon}$ é auto-adjunto e $\tilde{M}_{\epsilon}^{2}=\tilde{M}_{\epsilon}$, segue que

$$
\begin{aligned}
\left(\tilde{M}_{\epsilon}(u \cdot \nabla u), \tilde{M}_{\epsilon} v\right)_{\epsilon} & =\left(u \cdot \nabla u, \tilde{M}_{\epsilon}\left(\tilde{M}_{\epsilon} v\right)\right)_{\epsilon} \\
& =\left(u \cdot \nabla u, \tilde{M}_{\epsilon} v\right)_{\epsilon} \\
& =b_{\epsilon}\left(u, u, \tilde{M}_{\epsilon} v\right)
\end{aligned}
$$

Usando o item (iv) do lema 2.1,

$$
\begin{aligned}
\left(\tilde{M}_{\epsilon}(u \cdot \nabla u), \tilde{M}_{\epsilon} v\right)_{\epsilon} & =b_{\epsilon}\left(\tilde{M}_{\epsilon} u, \tilde{M}_{\epsilon} u, \tilde{M}_{\epsilon} v\right) \\
& +b_{\epsilon}\left(\tilde{N}_{\epsilon} u, \tilde{N}_{\epsilon} u, \tilde{M}_{\epsilon} v\right)
\end{aligned}
$$

Ainda,

$$
\begin{gathered}
-\mu\left(\Delta \tilde{M}_{\epsilon} u, \tilde{M}_{\epsilon} v\right)_{\epsilon}=\mu\left(\nabla \tilde{M}_{\epsilon} u, \nabla \tilde{M}_{\epsilon} v\right)_{\epsilon}= \\
\mu\left(A_{\epsilon}^{1 / 2} \tilde{M}_{\epsilon} u, A_{\epsilon}^{1 / 2} \tilde{M}_{\epsilon} v\right)_{\epsilon} .
\end{gathered}
$$

Além disso,

$$
\left(\nabla^{\prime} \tilde{M}_{\epsilon} p, \tilde{M}_{\epsilon} v\right)_{\epsilon}=0
$$

Substituindo (20), (21), (22) e (23) em (19), obtem-se a seguinte formulação fraca para $\tilde{M}_{\epsilon}$

$$
\frac{d}{d t}\left(\tilde{M}_{\epsilon} u, \tilde{M}_{\epsilon} v\right)_{\epsilon}+\mu\left(A_{\epsilon}^{1 / 2} \tilde{M}_{\epsilon} u, A_{\epsilon}^{1 / 2} \tilde{M}_{\epsilon} v\right)_{\epsilon}+
$$




$$
\begin{array}{r}
b_{\epsilon}\left(\tilde{M}_{\epsilon} u, \tilde{M}_{\epsilon} u, \tilde{M}_{\epsilon} v\right)+b_{\epsilon}\left(\tilde{N}_{\epsilon} u, \tilde{N}_{\epsilon} u, \tilde{M}_{\epsilon} v\right)= \\
\left(\tilde{M}_{\epsilon} f, \tilde{M}_{\epsilon} v\right)_{\epsilon} .
\end{array}
$$

Para o operador $\tilde{N}_{\epsilon}$, procede-se analogamente, aplicando $\tilde{N}_{\epsilon}$ à forma vetorial das equações de NavierStokes, multiplicando por $\tilde{N}_{\epsilon} v$ e integrando em $\Omega_{\epsilon}$. Deste modo, ao aplicar o item (iv) do lema 2.1, temse que

$$
\begin{aligned}
\left(\tilde{N}_{\epsilon}(u \cdot \nabla u), \tilde{N}_{\epsilon} v\right)_{\epsilon} & =\left(u \cdot \nabla u, \tilde{N}_{\epsilon} v\right)_{\epsilon} \\
& =b_{\epsilon}\left(u, u, \tilde{N}_{\epsilon} v\right) \\
& =b_{\epsilon}\left(\tilde{N}_{\epsilon} u, \tilde{M}_{\epsilon} u, \tilde{N}_{\epsilon} v\right) \\
& +b_{\epsilon}\left(\tilde{M}_{\epsilon} u, \tilde{N}_{\epsilon} u, \tilde{N}_{\epsilon} v\right) \\
& +b_{\epsilon}\left(\tilde{N}_{\epsilon} u, \tilde{N}_{\epsilon} u, \tilde{N}_{\epsilon} v\right) .
\end{aligned}
$$

Assim, conclui-se a formulação fraca para o operador $\tilde{N}_{\epsilon}$ dada por

$$
\begin{gathered}
\frac{d}{d t}\left(\tilde{N}_{\epsilon} u, \tilde{N}_{\epsilon} v\right)_{\epsilon}+\mu\left(A_{\epsilon}^{1 / 2} \tilde{N}_{\epsilon} u, A_{\epsilon}^{1 / 2} \tilde{N}_{\epsilon} v\right)_{\epsilon}+ \\
b_{\epsilon}\left(\tilde{M}_{\epsilon} u, \tilde{N}_{\epsilon} u, \tilde{N}_{\epsilon} v\right)+b_{\epsilon}\left(\tilde{N}_{\epsilon} u, \tilde{M}_{\epsilon} u, \tilde{N}_{\epsilon} v\right)+ \\
b_{\epsilon}\left(\tilde{N}_{\epsilon} u, \tilde{N}_{\epsilon} u, \tilde{N}_{\epsilon} v\right)=\left(\tilde{N}_{\epsilon} f, \tilde{N}_{\epsilon} v\right)_{\epsilon} .
\end{gathered}
$$

Para facilitar as expressões calculadas nas estimativas, utiliza-se, no que segue, as seguintes notações:
(i) $a_{0}(\epsilon)=\left|A_{\epsilon}^{1 / 2} \tilde{M}_{\epsilon} u_{0}\right|_{\epsilon}$;
(ii) $b_{0}(\epsilon)=\left|A_{\epsilon}^{1 / 2} \tilde{N}_{\epsilon} u_{0}\right|_{\epsilon}$;
(iii) $\alpha(\epsilon)=\left|\tilde{M}_{\epsilon} f\right|_{\epsilon}$;
(iv) $\beta(\epsilon)=\left|\tilde{N}_{\epsilon} f\right|_{\epsilon^{\prime}}$,
(v) $R_{0}^{2}(\epsilon)=a_{0}^{2}(\epsilon)+b_{0}^{2}(\epsilon)+\alpha^{2}(\epsilon)+\beta^{2}(\epsilon)$.

Observe que, de acordo com (ii) do lema 2.1,

$$
\begin{aligned}
\left|u_{0}\right|_{D\left(A_{\epsilon}^{1 / 2}\right)}^{2} & =\left|A_{\epsilon}^{1 / 2} u_{0}\right|_{\epsilon}^{2} \\
& =\left|\tilde{M}_{\epsilon} A_{\epsilon}^{1 / 2} u_{0}\right|_{\epsilon}^{2}+\left|\tilde{N}_{\epsilon} A_{\epsilon}^{1 / 2} u_{0}\right|_{\epsilon}^{2} \\
& =\left|A_{\epsilon}^{1 / 2} \tilde{M}_{\epsilon} u_{0}\right|_{\epsilon}^{2}+\left|A_{\epsilon}^{1 / 2} \tilde{N}_{\epsilon} u_{0}\right|_{\epsilon}^{2} \\
& =a_{0}^{2}(\epsilon)+b_{0}^{2}(\epsilon)
\end{aligned}
$$

$\mathrm{e}$

$$
|f|_{\epsilon}^{2}=\left|\tilde{M}_{\epsilon} f\right|_{\epsilon}^{2}+\left|\tilde{N}_{\epsilon} f\right|_{\epsilon}^{2}=\alpha^{2}(\epsilon)+\beta^{2}(\epsilon),
$$

ou seja,

$$
R_{0}^{2}(\epsilon)=\left|A_{\epsilon}^{1 / 2} u_{0}\right|_{\epsilon}^{2}+|f|_{\epsilon}^{2} .
$$

Agora, dado $\sigma>1$, como consequência do teorema 3.1, tem-se que existe $T^{\sigma}(\epsilon)>0$ tal que, para todo $t \in\left[0, T^{\sigma}(\epsilon)\right)$,

$$
\left|A_{\epsilon}^{1 / 2} u(t)\right|_{\epsilon}^{2} \leq \sigma R_{0}^{2}(\epsilon) .
$$

Aqui, $\left[0, T^{\sigma}(\epsilon)\right)$ é o intervalo máximo onde a desigualdade acima corresponde. Vale notar que se $T^{\sigma}(\epsilon)<\infty$, então

$$
\left|A_{\epsilon}^{1 / 2} u\left(T^{\sigma}(\epsilon)\right)\right|_{\epsilon}^{2}=\sigma R_{0}^{2}(\epsilon) .
$$

Com efeito, caso não valesse a igualdade, teria uma contradição com o fato de $T^{\sigma}(\epsilon)$ ser o tempo máximo.

Apresentamos agora dois lemas com as estimativas para os operadores $\tilde{N}_{\epsilon}$ e $\tilde{M}_{\epsilon}$, fundamentais para prova dos teoremas de existência. O resultado a seguir independe da condição de fronteira considerada.

Lema 3.1 (Estimativas para $\tilde{N}_{\epsilon} u$ ). Suponha que $0<q<\frac{1}{2}$ $e$

$$
\lim _{\epsilon \rightarrow 0} \epsilon^{2 q}\left(\left|A_{\epsilon}^{1 / 2} u_{0}\right|_{\epsilon}^{2}+|f|_{\epsilon}^{2}\right)=0 .
$$

Então, existe $\epsilon_{1}=\epsilon_{1}(\mu)>0$ tal que para todo $\epsilon, 0<$ $\epsilon \leq \epsilon_{1}$, existem $T^{\sigma}(\epsilon)>0$ e uma constante positiva $c_{5}(\mu)$, independente de $\epsilon$, de modo que para $\epsilon, 0<\epsilon \leq \epsilon_{1} e 0<$ $t<T^{\sigma}(\epsilon)$, tem-se:

$$
\begin{aligned}
& \text { (i) }\left|A_{\epsilon}^{1 / 2} \tilde{N}_{\epsilon} u(t)\right|_{\epsilon}^{2} \leq b_{0}^{2}(\epsilon) e^{-\frac{\mu t}{2 \epsilon^{2}}}+\frac{2 \epsilon^{2}}{\mu^{2}} \beta^{2}(\epsilon) ; \\
& \text { (ii) } \int_{0}^{t}\left|A_{\epsilon} \tilde{N}_{\epsilon} u(s)\right|_{\epsilon}^{2} d s \leq \frac{2}{\mu} b_{0}^{2}(\epsilon)+\frac{2}{\mu^{2}} t \beta^{2}(\epsilon) ; \\
& \text { (iii) } \int_{0}^{t}\left|A_{\epsilon}^{1 / 2} \tilde{N}_{\epsilon} u(s)\right|_{\epsilon}^{3}\left|A_{\epsilon} \tilde{N}_{\epsilon} u(s)\right|_{\epsilon} d s \leq \\
& c_{5}(\mu) \epsilon\left(b_{0}^{2}(\epsilon)+\epsilon^{2} \beta^{2}(\epsilon)\right)\left(b_{0}^{2}(\epsilon)+t \beta^{2}(\epsilon)\right) .
\end{aligned}
$$

Demonstração: Da formulação fraca para $\tilde{N}_{\epsilon}$, substituindo $v$ por $A_{\epsilon} u$, tem-se

$$
\begin{gathered}
\frac{d}{d t}\left(\tilde{N}_{\epsilon} u, \tilde{N}_{\epsilon} A_{\epsilon} u\right)_{\epsilon}+\mu\left(A_{\epsilon}^{1 / 2} \tilde{N}_{\epsilon} u, A_{\epsilon}^{1 / 2} \tilde{N}_{\epsilon} A_{\epsilon} u\right)_{\epsilon}+ \\
b_{\epsilon}\left(\tilde{M}_{\epsilon} u, \tilde{N}_{\epsilon} u, \tilde{N}_{\epsilon} A_{\epsilon} u\right)+b_{\epsilon}\left(\tilde{N}_{\epsilon} u, \tilde{M}_{\epsilon} u, \tilde{N}_{\epsilon} A_{\epsilon} u\right)+ \\
b_{\epsilon}\left(\tilde{N}_{\epsilon} u, \tilde{N}_{\epsilon} u, \tilde{N}_{\epsilon} A_{\epsilon} u\right)=\left(\tilde{N}_{\epsilon} f, \tilde{N}_{\epsilon} A_{\epsilon} u\right)_{\epsilon} .
\end{gathered}
$$

Observe que, pela comutatividade do operador de Stokes com $\tilde{N}_{\epsilon}$,

$$
\begin{aligned}
\left(\tilde{N}_{\epsilon} u, \tilde{N}_{\epsilon} A_{\epsilon} u\right)_{\epsilon} & =\left(\tilde{N}_{\epsilon} u, A_{\epsilon} \tilde{N}_{\epsilon} u\right)_{\epsilon}=\left(\left(\tilde{N}_{\epsilon} u, \tilde{N}_{\epsilon} u\right)\right)_{\epsilon} \\
& =\left(A_{\epsilon}^{1 / 2} \tilde{N}_{\epsilon} u, A_{\epsilon}^{1 / 2} \tilde{N}_{\epsilon} u\right)_{\epsilon} \\
& =\left|A_{\epsilon}^{1 / 2} \tilde{N}_{\epsilon} u\right|_{\epsilon}^{\prime}
\end{aligned}
$$

Logo,

$$
\frac{d}{d t}\left(\tilde{N}_{\epsilon} u, \tilde{N}_{\epsilon} A_{\epsilon} u\right)_{\epsilon}=\frac{d}{d t}\left|A_{\epsilon}^{1 / 2} \tilde{N}_{\epsilon} u\right|_{\epsilon}^{2} .
$$


Mas

$$
\begin{array}{r}
\frac{d}{d t}\left(\tilde{N}_{\epsilon} u, \tilde{N}_{\epsilon} A_{\epsilon} u\right)_{\epsilon}= \\
\left(\frac{d}{d t} \tilde{N}_{\epsilon} u, \tilde{N}_{\epsilon} A_{\epsilon} u\right)_{\epsilon}+\left(\tilde{N}_{\epsilon} u, \frac{d}{d t} \tilde{N}_{\epsilon} A_{\epsilon} u\right)_{\epsilon}= \\
\left(\frac{d}{d t} \tilde{N}_{\epsilon} u, \tilde{N}_{\epsilon} A_{\epsilon} u\right)_{\epsilon}+\left(\tilde{N}_{\epsilon} u, \frac{d}{d t} A_{\epsilon} \tilde{N}_{\epsilon} u\right)_{\epsilon}= \\
\left(\frac{d}{d t} \tilde{N}_{\epsilon} u, \tilde{N}_{\epsilon} A_{\epsilon} u\right)_{\epsilon}+\left(\tilde{N}_{\epsilon} u, A_{\epsilon}\left(\frac{d}{d t} \tilde{N}_{\epsilon} u\right)\right)_{\epsilon}= \\
\left(\frac{d}{d t} \tilde{N}_{\epsilon} u, \tilde{N}_{\epsilon} A_{\epsilon} u\right)_{\epsilon}+\left(A_{\epsilon} \tilde{N}_{\epsilon} u, \frac{d}{d t} \tilde{N}_{\epsilon} u\right)_{\epsilon}= \\
2\left(\frac{d}{d t} \tilde{N}_{\epsilon} u, \tilde{N}_{\epsilon} A_{\epsilon} u\right)_{\epsilon} \cdot
\end{array}
$$

Assim, de (27),

$$
\begin{aligned}
\left(\frac{d}{d t} \tilde{N}_{\epsilon} u, \tilde{N}_{\epsilon} A_{\epsilon} u\right)_{\epsilon} & =\frac{1}{2} \frac{d}{d t}\left(\tilde{N}_{\epsilon} u, \tilde{N}_{\epsilon} A_{\epsilon} u\right)_{\epsilon} \\
& =\frac{1}{2} \frac{d}{d t}\left|A_{\epsilon}^{1 / 2} \tilde{N}_{\epsilon} u\right|_{\epsilon}^{2} .
\end{aligned}
$$

Também,

$$
\begin{aligned}
& \mu\left(A_{\epsilon}^{1 / 2} \tilde{N}_{\epsilon} u, A_{\epsilon}^{1 / 2} \tilde{N}_{\epsilon} A_{\epsilon} u\right)_{\epsilon}= \\
& \mu\left(A_{\epsilon}^{1 / 2} \tilde{N}_{\epsilon} u, A_{\epsilon}^{1 / 2} A_{\epsilon} \tilde{N}_{\epsilon} u\right)_{\epsilon}= \\
& \mu\left(A_{\epsilon} \tilde{N}_{\epsilon} u, A_{\epsilon} \tilde{N}_{\epsilon} u\right)_{\epsilon}= \\
& \mu\left|A_{\epsilon} \tilde{N}_{\epsilon} u\right|_{\epsilon}^{2} .
\end{aligned}
$$

Além disso, pela desigualdade de Cauchy-Schwarz,

$$
\begin{aligned}
\left(\tilde{N}_{\epsilon} f, \tilde{N}_{\epsilon} A_{\epsilon} u\right)_{\epsilon} & =\left(\tilde{N}_{\epsilon} f, A_{\epsilon} \tilde{N}_{\epsilon} u\right)_{\epsilon} \\
& \leq\left|\tilde{N}_{\epsilon} f\right|_{\epsilon}\left|A_{\epsilon} \tilde{N}_{\epsilon} u\right|_{\epsilon} \\
& \leq \frac{\delta^{2}}{2}\left|\tilde{N}_{\epsilon} f\right|_{\epsilon}^{2}+\frac{1}{2 \delta^{2}}\left|A_{\epsilon} \tilde{N}_{\epsilon} u\right|_{\epsilon}^{2} .
\end{aligned}
$$

A última desigualdade acima vem de $2 a b \leq a^{2}+b^{2}$, tomando $a=\delta\left|\tilde{N}_{\epsilon} f\right|_{\epsilon}$ e $b=\delta^{-1}\left|A_{\epsilon} \tilde{N}_{\epsilon} u\right|_{\epsilon^{\prime}}$ onde $\delta>0$ é um número real. Escolhendo $\frac{1}{2 \delta^{2}}=\frac{\mu}{2}$, tem-se $\delta^{2}=\mu^{-1}$. Daí,

$$
\left(\tilde{N}_{\epsilon} f, \tilde{N}_{\epsilon} A_{\epsilon} u\right)_{\epsilon} \leq \frac{1}{2 \mu}\left|\tilde{N}_{\epsilon} f\right|_{\epsilon}^{2}+\frac{\mu}{2}\left|A_{\epsilon} \tilde{N}_{\epsilon} u\right|_{\epsilon}^{2}
$$

Pelas estimativas do Lema 2.4,

$$
\begin{aligned}
& b_{\epsilon}\left(\tilde{M}_{\epsilon} u, \tilde{N}_{\epsilon} u, \tilde{N}_{\epsilon} A_{\epsilon} u\right)+b_{\epsilon}\left(\tilde{N}_{\epsilon} u, \tilde{M}_{\epsilon} u, \tilde{N}_{\epsilon} A_{\epsilon} u\right) \\
+ & b_{\epsilon}\left(\tilde{N}_{\epsilon} u, \tilde{N}_{\epsilon} u, \tilde{N}_{\epsilon} A_{\epsilon} u\right) \\
\leq & c_{4} \epsilon^{q} \|\left.\tilde{M}_{\epsilon} u\right|_{\epsilon}\left|A_{\epsilon} \tilde{N}_{\epsilon} u\right|_{\epsilon}\left|A_{\epsilon} \tilde{N}_{\epsilon} u\right|_{\epsilon} \\
+ & c_{4} \epsilon^{1 / 2} \|\left.\tilde{M}_{\epsilon} u\right|_{\epsilon}\left|A_{\epsilon} \tilde{N}_{\epsilon} u\right|_{\epsilon}\left|A_{\epsilon} \tilde{N}_{\epsilon} u\right|_{\epsilon} \\
+ & c_{4} \epsilon^{1 / 2}|| \tilde{N}_{\epsilon} u \|_{\epsilon}\left|A_{\epsilon} \tilde{N}_{\epsilon} u\right|_{\epsilon}\left|A_{\epsilon} \tilde{N}_{\epsilon} u\right|_{\epsilon} \\
= & \left.c_{4} \epsilon^{q}\left\|\left.\tilde{M}_{\epsilon} u\right|_{\epsilon}\left|A_{\epsilon} \tilde{N}_{\epsilon} u\right|_{\epsilon}^{2}+c_{4} \epsilon^{1 / 2}\right\| \tilde{M}_{\epsilon} u\right|_{\epsilon}\left|A_{\epsilon} \tilde{N}_{\epsilon} u\right|_{\epsilon}^{2}
\end{aligned}
$$

$$
+c_{4} \epsilon^{1 / 2}|| \tilde{N}_{\epsilon} u\|\|_{\epsilon}\left|A_{\epsilon} \tilde{N}_{\epsilon} u\right|_{\epsilon}^{2} .
$$

Note que

$$
\begin{aligned}
\left\|\tilde{M}_{\epsilon} u\right\|_{\epsilon}^{2} & =\left(\left(\tilde{M}_{\epsilon} u, \tilde{M}_{\epsilon} u\right)\right)_{\epsilon}=\left(A_{\epsilon} \tilde{M}_{\epsilon} u, \tilde{M}_{\epsilon} u\right)_{\epsilon} \\
& =\left(A_{\epsilon}^{1 / 2} \tilde{M}_{\epsilon} u, A_{\epsilon}^{1 / 2} \tilde{M}_{\epsilon} u\right)_{\epsilon} \\
& =\left|A_{\epsilon}^{1 / 2} \tilde{M}_{\epsilon} u\right|_{\epsilon}^{2},
\end{aligned}
$$

ou seja,

$$
\left\|\tilde{M}_{\epsilon} u\right\|_{\epsilon}=\left|A_{\epsilon}^{1 / 2} \tilde{M}_{\epsilon} u\right|_{\epsilon} .
$$

Analogamente,

$$
\left\|\tilde{N}_{\epsilon} u\right\|_{\epsilon}=\left|A_{\epsilon}^{1 / 2} \tilde{N}_{\epsilon} u\right|_{\epsilon},
$$

e, com isso,

$$
\begin{aligned}
& b_{\epsilon}\left(\tilde{M}_{\epsilon} u, \tilde{N}_{\epsilon} u, \tilde{N}_{\epsilon} A_{\epsilon} u\right)+b_{\epsilon}\left(\tilde{N}_{\epsilon} u, \tilde{M}_{\epsilon} u, \tilde{N}_{\epsilon} A_{\epsilon} u\right) \\
+ & b_{\epsilon}\left(\tilde{N}_{\epsilon} u, \tilde{N}_{\epsilon} u, \tilde{N}_{\epsilon} A_{\epsilon} u\right) \\
\leq & c_{4} \epsilon^{q}\left|A_{\epsilon}^{1 / 2} \tilde{M}_{\epsilon} u\right|_{\epsilon}\left|A_{\epsilon} \tilde{N}_{\epsilon} u\right|_{\epsilon}^{2} \\
+ & c_{4} \epsilon^{1 / 2}\left(\left|A_{\epsilon}^{1 / 2} \tilde{M}_{\epsilon} u\right|_{\epsilon}+\left|A_{\epsilon}^{1 / 2} \tilde{N}_{\epsilon} u\right|_{\epsilon}\right)\left|A_{\epsilon} \tilde{N}_{\epsilon} u\right|_{\epsilon}^{2} .
\end{aligned}
$$

Temos, portanto, a seguinte estimativa

$$
\begin{aligned}
& \frac{1}{2} \frac{d}{d t}\left|A_{\epsilon}^{1 / 2} \tilde{N}_{\epsilon} u\right|_{\epsilon}^{2}+\mu\left|A_{\epsilon} \tilde{N}_{\epsilon} u\right|_{\epsilon}^{2} \leq \\
& \frac{1}{2 \mu}\left|\tilde{N}_{\epsilon} f\right|_{\epsilon}^{2}+\frac{\mu}{2}\left|A_{\epsilon} \tilde{N}_{\epsilon} u\right|_{\epsilon}^{2}+ \\
& {\left[c_{4} \epsilon^{q}\left|A_{\epsilon}^{1 / 2} \tilde{M}_{\epsilon} u\right|_{\epsilon}\left|A_{\epsilon} \tilde{N}_{\epsilon} u\right|_{\epsilon}^{2}+\right.} \\
& \left.c_{4} \epsilon^{1 / 2}\left(\left|A_{\epsilon}^{1 / 2} \tilde{M}_{\epsilon} u\right|_{\epsilon}+\left|A_{\epsilon}^{1 / 2} \tilde{N}_{\epsilon} u\right|_{\epsilon}\right)\left|A_{\epsilon} \tilde{N}_{\epsilon} u\right|_{\epsilon}^{2}\right] \text {. }
\end{aligned}
$$

Como $0<\epsilon<1$ e $0<q<\frac{1}{2}$, pode-se afirmar que $\epsilon^{1 / 2}<\epsilon^{q}$. Assim, somando $c_{4} \epsilon^{q}\left|A_{\epsilon}^{1 / 2} \tilde{N}_{\epsilon} u\right|_{\epsilon}\left|A_{\epsilon} \tilde{N}_{\epsilon} u\right|_{\epsilon^{\prime}}^{2}$ o qual é um valor positivo, na última expressão acima, obtem-se

$$
\begin{aligned}
\frac{d}{d t}\left|A_{\epsilon}^{1 / 2} \tilde{N}_{\epsilon} u\right|_{\epsilon}^{2} & +\left[\mu-4 c_{4} \epsilon^{q}\left(\left|A_{\epsilon}^{1 / 2} \tilde{M}_{\epsilon} u\right|_{\epsilon}\right.\right. \\
& \left.\left.+\left|A_{\epsilon}^{1 / 2} \tilde{N}_{\epsilon} u\right|_{\epsilon}\right)\right]\left|A_{\epsilon} \tilde{N}_{\epsilon} u\right|_{\epsilon}^{2} \\
& \leq \frac{1}{\mu}\left|\tilde{N}_{\epsilon} f\right|_{\epsilon}^{2} .
\end{aligned}
$$

Observe que, do item (ii) do Lema 2.1,

$$
\begin{aligned}
\left|A_{\epsilon}^{1 / 2} u(t)\right|_{\epsilon}^{2} & =\left|\tilde{M}_{\epsilon} A_{\epsilon}^{1 / 2} u(t)\right|_{\epsilon}^{2}+\left|\tilde{N}_{\epsilon} A_{\epsilon}^{1 / 2} u(t)\right|_{\epsilon}^{2} \\
& =\left|A_{\epsilon}^{1 / 2} \tilde{M}_{\epsilon} u(t)\right|_{\epsilon}^{2}+\left|A_{\epsilon}^{1 / 2} \tilde{N}_{\epsilon} u(t)\right|_{\epsilon}^{2}
\end{aligned}
$$

para $t \in\left[0, T^{\sigma}(\epsilon)\right)$ e, além disso,

$$
\left|A_{\epsilon}^{1 / 2} \tilde{M}_{\epsilon} u(t)\right|_{\epsilon}+\left|A_{\epsilon}^{1 / 2} \tilde{N}_{\epsilon} u(t)\right|_{\epsilon} \leq \sqrt{2}\left|A_{\epsilon}^{1 / 2} u(t)\right|_{\epsilon} .
$$


Mas, de (25), temos

$$
\left|A_{\epsilon}^{1 / 2} \tilde{M}_{\epsilon} u(t)\right|_{\epsilon}+\left|A_{\epsilon}^{1 / 2} \tilde{N}_{\epsilon} u(t)\right|_{\epsilon} \leq \sqrt{2} \sqrt{\sigma} R_{0}(\epsilon) .
$$

Assim,

$$
\begin{gathered}
\mu-4 c_{4} \epsilon^{q}\left(\left|A_{\epsilon}^{1 / 2} \tilde{M}_{\epsilon} u\right|_{\epsilon}+\left|A_{\epsilon}^{1 / 2} \tilde{N}_{\epsilon} u\right|_{\epsilon}\right) \geq \\
\mu-4 c_{4} \epsilon^{q} \sqrt{2} \sqrt{\sigma} R_{0}(\epsilon),
\end{gathered}
$$

isto é,

$$
\begin{array}{r}
\frac{d}{d t}\left|A_{\epsilon}^{1 / 2} \tilde{N}_{\epsilon} u\right|_{\epsilon}^{2}+\left[\mu-4 c_{4} \epsilon^{q} \sqrt{2 \sigma} R_{0}(\epsilon)\right]\left|A_{\epsilon} \tilde{N}_{\epsilon} u\right|_{\epsilon}^{2} \leq \\
\frac{1}{\mu}\left|\tilde{N}_{\epsilon} f\right|_{\epsilon}^{2} .
\end{array}
$$

A partir deste momento, usando a hipótese

$$
\lim _{\epsilon \rightarrow 0} \epsilon^{2 q}\left(\left|A_{\epsilon}^{1 / 2} u_{0}\right|_{\epsilon}^{2}+|f|_{\epsilon}^{2}\right)=\lim _{\epsilon \rightarrow 0} \epsilon^{2 q} R_{0}^{2}(\epsilon)=0,
$$

pode-se afirmar que

$$
\lim _{\epsilon \rightarrow 0}\left(\mu-4 c_{4} \epsilon^{q} \sqrt{2 \sigma} R_{0}(\epsilon)\right)=\mu>0,
$$

ou seja, existe um $\epsilon_{1}>0$ tal que para todo $0<\epsilon<\epsilon_{1}$,

$$
\mu-4 c_{4} \epsilon^{q} \sqrt{2 \sigma} R_{0}(\epsilon) \leq \frac{\mu}{2}
$$

Substituindo em (29),

$$
\frac{d}{d t}\left|A_{\epsilon}^{1 / 2} \tilde{N}_{\epsilon} u\right|_{\epsilon}^{2}+\frac{\mu}{2}\left|A_{\epsilon} \tilde{N}_{\epsilon} u\right|_{\epsilon}^{2} \leq \frac{1}{\mu}\left|\tilde{N}_{\epsilon} f\right|_{\epsilon}^{2},
$$

para todo $0<\epsilon<\epsilon_{1}$ e $0 \leq t<T^{\sigma}(\epsilon)$.

Pela desigualdade de Cauchy-Schwarz, tem-se

$$
\left|A_{\epsilon}^{1 / 2} \tilde{N}_{\epsilon} u\right|_{\epsilon}^{2} \leq \epsilon^{2}\left|A_{\epsilon} \tilde{N}_{\epsilon} u\right|_{\epsilon}^{2}
$$

e portanto,

$$
\frac{d}{d t}\left|A_{\epsilon}^{1 / 2} \tilde{N}_{\epsilon} u\right|_{\epsilon}^{2}+\frac{\mu}{2 \epsilon^{2}}\left|A_{\epsilon}^{1 / 2} \tilde{N}_{\epsilon} u\right|_{\epsilon}^{2} \leq \frac{1}{\mu}\left|\tilde{N}_{\epsilon} f\right|_{\epsilon}^{2} .
$$

Agora, aplicando a desigualdade de Gronwall, obtem-se

$$
\begin{aligned}
\left|A_{\epsilon}^{1 / 2} \tilde{N}_{\epsilon} u\right|_{\epsilon}^{2} & \leq e^{-\frac{\mu t}{2 \epsilon^{2}}} b_{0}^{2}(\epsilon)+\frac{e^{-\frac{\mu t}{2 \epsilon^{2}}}}{\mu} \int_{0}^{t} \beta^{2}(\epsilon) d s \\
& \leq e^{-\frac{\mu t}{2 \epsilon^{2}}} b_{0}^{2}(\epsilon)+\frac{e^{-\frac{\mu t}{2 \epsilon^{2}}}}{\mu} t \beta^{2}(\epsilon)
\end{aligned}
$$

Para algum $t, \operatorname{com} t \leq \frac{2 \epsilon^{2}}{\mu} e^{\frac{\mu t}{2 \epsilon^{2}}}$, vale que

$$
\frac{e^{-\frac{\mu t}{2 \epsilon^{2}}}}{\mu} t \beta^{2}(\epsilon) \leq \frac{2 \epsilon^{2}}{\mu^{2}} \beta^{2}(\epsilon)
$$

e, substituindo em (32),

$$
\left|A_{\epsilon}^{1 / 2} \tilde{N}_{\epsilon} u\right|_{\epsilon}^{2} \leq b_{0}^{2}(\epsilon) e^{-\frac{\mu t}{2 \epsilon^{2}}}+\frac{2 \epsilon^{2}}{\mu^{2}} \beta^{2}(\epsilon),
$$

o que prova o item (i) do lema.

Integrando de 0 à $t$ a expressão em (31), tem-se

$$
\begin{gathered}
\left|A_{\epsilon}^{1 / 2} \tilde{N}_{\epsilon} u(t)\right|_{\epsilon}^{2}+\frac{\mu}{2 \epsilon^{2}} \int_{0}^{t}\left|A_{\epsilon}^{1 / 2} \tilde{N}_{\epsilon} u(s)\right|_{\epsilon}^{2} d s \leq \\
b_{0}^{2}(\epsilon)+\frac{1}{\mu} t \beta^{2}(\epsilon) .
\end{gathered}
$$

Como

$$
\begin{aligned}
& \frac{\mu}{2 \epsilon^{2}} \int_{0}^{t}\left|A_{\epsilon}^{1 / 2} \tilde{N}_{\epsilon} u(s)\right|_{\epsilon}^{2} d s \leq\left|A_{\epsilon}^{1 / 2} \tilde{N}_{\epsilon} u(t)\right|_{\epsilon}^{2}+ \\
& \frac{\mu}{2 \epsilon^{2}} \int_{0}^{t}\left|A_{\epsilon}^{1 / 2} \tilde{N}_{\epsilon} u(s)\right|_{\epsilon}^{2} d s,
\end{aligned}
$$

obtem-se

$$
\frac{\mu}{2 \epsilon^{2}} \int_{0}^{t}\left|A_{\epsilon}^{1 / 2} \tilde{N}_{\epsilon} u(s)\right|_{\epsilon}^{2} d s \leq b_{0}^{2}(\epsilon)+\frac{1}{\mu} t \beta^{2}(\epsilon),
$$

ou seja,

$$
\int_{0}^{t}\left|A_{\epsilon}^{1 / 2} \tilde{N}_{\epsilon} u(s)\right|_{\epsilon}^{2} d s \leq \frac{2 \epsilon^{2}}{\mu} b_{0}^{2}(\epsilon)+\frac{2 \epsilon^{2}}{\mu^{2}} t \beta^{2}(\epsilon),
$$

para todo $0<\epsilon \leq \epsilon_{1}$ e $0<t<T^{\sigma}(\epsilon)$.

Agora, integrando (30) de 0 à $t$,

$$
\begin{gathered}
\left|A_{\epsilon}^{1 / 2} \tilde{N}_{\epsilon} u(t)\right|_{\epsilon}^{2}+\frac{\mu}{2} \int_{0}^{t}\left|A_{\epsilon} \tilde{N}_{\epsilon} u(s)\right|_{\epsilon}^{2} d s \leq \\
b_{0}^{2}(\epsilon)+\frac{1}{\mu} t \beta^{2}(\epsilon) .
\end{gathered}
$$

Daí,

$$
\frac{\mu}{2} \int_{0}^{t}\left|A_{\epsilon} \tilde{N}_{\epsilon} u(s)\right|_{\epsilon}^{2} d s \leq b_{0}^{2}(\epsilon)+\frac{1}{\mu} t \beta^{2}(\epsilon)
$$

ou seja,

$$
\int_{0}^{t}\left|A_{\epsilon} \tilde{N}_{\epsilon} u(s)\right|_{\epsilon}^{2} d s \leq \frac{2}{\mu} b_{0}^{2}(\epsilon)+\frac{2}{\mu^{2}} t \beta^{2}(\epsilon),
$$

para todo $0<\epsilon \leq \epsilon_{1}$ e $0<t<T^{\sigma}(\epsilon)$, provando assim o item (ii) do lema.

Aplicando a desigualdade de Hölder com $p=q=2$ para as funções $u=\left|A_{\epsilon}^{1 / 2} \tilde{N}_{\epsilon} u\right|_{\epsilon}^{3}$ e $v=\left|A_{\epsilon} \tilde{N}_{\epsilon} u\right|_{\epsilon^{\prime}}$, temos

$$
\underbrace{\int_{0}^{t}\left|A_{\epsilon}^{1 / 2} \tilde{N}_{\epsilon} u(s)\right|_{\epsilon}^{3}\left|A_{\epsilon} \tilde{N}_{\epsilon} u(s)\right|_{\epsilon} d s}_{=\Psi} \leq
$$

$\left(\int_{0}^{t}\left|A_{\epsilon}^{1 / 2} \tilde{N}_{\epsilon} u(s)\right|_{\epsilon}^{6} d s\right)^{1 / 2}\left(\int_{0}^{t}\left|A_{\epsilon} \tilde{N}_{\epsilon} u(s)\right|_{\epsilon}^{2} d s\right)^{1 / 2}$ 
Observe que

$$
\begin{array}{r}
\left(\int_{0}^{t}\left|A_{\epsilon}^{1 / 2} \tilde{N}_{\epsilon} u(s)\right|_{\epsilon}^{6} d s\right)^{1 / 2} \leq \\
\sup _{0 \leq s \leq t}\left|A_{\epsilon}^{1 / 2} \tilde{N}_{\epsilon} u(s)\right|_{\epsilon}^{2}\left(\int_{0}^{t}\left|A_{\epsilon}^{1 / 2} \tilde{N}_{\epsilon} u(s)\right|_{\epsilon}^{2} d s\right)^{1 / 2}
\end{array}
$$

Portanto,

$$
\begin{aligned}
\Psi \leq & \sup _{0 \leq s \leq t}\left|A_{\epsilon}^{1 / 2} \tilde{N}_{\epsilon} u(s)\right|_{\epsilon}^{2}\left(\int_{0}^{t}\left|A_{\epsilon}^{1 / 2} \tilde{N}_{\epsilon} u(s)\right|_{\epsilon}^{2} d s\right)^{1 / 2} \\
& \cdot\left(\int_{0}^{t}\left|A_{\epsilon} \tilde{N}_{\epsilon} u(s)\right|_{\epsilon} d s\right)^{1 / 2}
\end{aligned}
$$

Note que é possível tomar o supremo da função $\left|A_{\epsilon}^{1 / 2} \tilde{N}_{\epsilon} u\right|_{\epsilon}{ }^{\prime}$, pois vale (33).

Substituindo as estimativas encontradas em (33), (34) e (35) na expressão acima, obtem-se

$$
\Psi \leq 4 \epsilon\left(b_{0}^{2}(\epsilon)+\frac{2 \epsilon^{2}}{\mu^{2}} \beta^{2}(\epsilon)\right)\left(\frac{1}{\mu} b_{0}^{2}(\epsilon)+\frac{1}{\mu^{2}} t \beta^{2}(\epsilon)\right) .
$$

Fazendo

$$
c_{5}(\mu)=\left[2 \max \left(1, \frac{1}{\mu^{2}}, \frac{1}{\mu}\right)\right]^{2},
$$

conclui-se que

$$
\Psi \leq c_{5}(\mu) \epsilon\left(b_{0}^{2}(\epsilon)+\epsilon^{2} \beta^{2}(\epsilon)\right)\left(b_{0}^{2}(\epsilon)+t \beta^{2}(\epsilon)\right)
$$

o que prova o item (iii) e, portanto, o lema está demonstrado.

Lema 3.2 (Estimativas para $\tilde{M}_{\epsilon} u$ ). Considerando as condições $(F F)$ e $(F P)$, suponha que $0<q<\frac{1}{2} e$

$$
\lim _{\epsilon \rightarrow 0} \epsilon^{2 q}\left(\left|A_{\epsilon}^{1 / 2} u_{0}\right|_{\epsilon}^{2}+|f|_{\epsilon}^{2}\right)=0 .
$$

Então, existe $\epsilon_{1}=\epsilon_{1}(\mu)>0$, tal que para todo $\epsilon$, com $0<\epsilon \leq \epsilon_{1}$, existem $T^{\sigma}(\epsilon)>0$ e uma constante positiva $c_{10}(\mu)$, independente de $\epsilon$, a partir dos quais vale:

(i) $\left|\tilde{M}_{\epsilon} u(t)\right|_{\epsilon}^{2} \leq a_{0}^{2}(\epsilon) e^{-\mu \lambda_{1} t}+\frac{\alpha^{2}(\epsilon)}{\mu^{2} \lambda_{1}^{3}}$ $+c_{6}(\mu) \epsilon R_{n}^{4}(\epsilon)(1+t) ;$

(ii) $\int_{0}^{t}\left|A_{\epsilon}^{1 / 2} \tilde{M}_{\epsilon} u(s)\right|_{\epsilon}^{2} d s \leq \frac{a_{0}^{2}(\epsilon)}{\mu \lambda_{1}}+\frac{2 \alpha^{2}(\epsilon) t}{\mu^{2} \lambda_{1}^{2}}$ $+c_{6}(\mu) \epsilon R_{n}^{4}(\epsilon)(1+t)$;

(iii) $\left|A_{\epsilon}^{1 / 2} \tilde{M}_{\epsilon} u(t)\right|_{\epsilon}^{2} \leq a_{0}^{2}(\epsilon) e^{-\mu \lambda_{1}^{2} t}+\frac{2 \alpha^{2}(\epsilon)}{\mu^{2} \lambda_{1}}+$ $c_{8}(\mu) \epsilon R_{n}^{4}(\epsilon)(1+t)$

$$
\text { (iv) } \begin{aligned}
& \int_{0}^{t}\left|A_{\epsilon} \tilde{M}_{\epsilon} u(s)\right|_{\epsilon}^{2} d s \leq \frac{2 a_{0}^{2}(\epsilon) t}{\mu}+ \\
& c_{8}(\mu) \epsilon R_{n}^{4}(\epsilon)(1+t) .
\end{aligned}
$$

Demonstração: Da formulação fraca para $\tilde{M}_{\epsilon}$, substituindo $v$ por $u$, tem-se

$$
\begin{array}{r}
\frac{d}{d t}\left(\tilde{M}_{\epsilon} u, \tilde{M}_{\epsilon} u\right)_{\epsilon}+\mu\left(A_{\epsilon}^{1 / 2} \tilde{M}_{\epsilon} u, A_{\epsilon}^{1 / 2} \tilde{M}_{\epsilon} u\right)_{\epsilon} \\
b_{\epsilon}\left(\tilde{M}_{\epsilon} u, \tilde{M}_{\epsilon} u, \tilde{M}_{\epsilon} u\right)+b_{\epsilon}\left(\tilde{N}_{\epsilon} u, \tilde{N}_{\epsilon} u, \tilde{M}_{\epsilon} u\right)= \\
\left(\tilde{M}_{\epsilon} f, \tilde{M}_{\epsilon} u\right)_{\epsilon}
\end{array}
$$

É possível afirmar (veja Temam (1984), Lema 1.3, pág. 162) que

$$
b_{\epsilon}\left(\tilde{M}_{\epsilon} u, \tilde{M}_{\epsilon} u, \tilde{M}_{\epsilon} u\right)=0,
$$

e, pela desigualdade de Cauchy-Schwarz,

$$
\left(\tilde{M}_{\epsilon} f, \tilde{M}_{\epsilon} u\right)_{\epsilon} \leq\left|\tilde{M}_{\epsilon} f\right|_{\epsilon}\left|\tilde{M}_{\epsilon} u\right|_{\epsilon}
$$

Assim como foi feito no lema anterior para $\tilde{N}_{\epsilon}$, o termo com a derivada em relação ao tempo é tal que, antes de substituir,

$$
\frac{d}{d t}\left(\tilde{M}_{\epsilon} u, \tilde{M}_{\epsilon} v\right)_{\epsilon}=\left(\frac{d}{d t} \tilde{M}_{\epsilon} u, \tilde{M}_{\epsilon} v\right)_{\epsilon},
$$

pois $v \in V_{\epsilon}$ e não depende de $t$. Mas,

$$
\begin{aligned}
\frac{d}{d t}\left(\tilde{M}_{\epsilon} u, \tilde{M}_{\epsilon} u\right)_{\epsilon} & =\left(\frac{d}{d t} \tilde{M}_{\epsilon} u, \tilde{M}_{\epsilon} u\right)_{\epsilon} \\
+\left(\tilde{M}_{\epsilon} u, \frac{d}{d t} \tilde{M}_{\epsilon} u\right)_{\epsilon} & =2\left(\frac{d}{d t} \tilde{M}_{\epsilon} u, \tilde{M}_{\epsilon} u\right)_{\epsilon}
\end{aligned}
$$

ou seja,

$$
\begin{aligned}
\frac{d}{d t}\left(\tilde{M}_{\epsilon} u, \tilde{M}_{\epsilon} u\right)_{\epsilon} & =\left(\frac{d}{d t} \tilde{M}_{\epsilon} u, \tilde{M}_{\epsilon} u\right)_{\epsilon} \\
& =\frac{1}{2} \frac{d}{d t}\left(\tilde{M}_{\epsilon} u, \tilde{M}_{\epsilon} u\right)_{\epsilon} \\
& =\frac{1}{2} \frac{d}{d t}\left|\tilde{M}_{\epsilon} u\right|_{\epsilon}^{2} .
\end{aligned}
$$

Ainda,

$$
\mu\left(A_{\epsilon}^{1 / 2} \tilde{M}_{\epsilon} u, A_{\epsilon}^{1 / 2} \tilde{M}_{\epsilon} u\right)_{\epsilon}=\mu\left|A_{\epsilon}^{1 / 2} \tilde{M}_{\epsilon} u\right|_{\epsilon}^{2} .
$$

Logo,

$$
\begin{array}{r}
\frac{1}{2} \frac{d}{d t}\left|\tilde{M}_{\epsilon} u\right|_{\epsilon}^{2}+\mu\left|A_{\epsilon}^{1 / 2} \tilde{M}_{\epsilon} u\right|_{\epsilon}^{2}+ \\
b_{\epsilon}\left(\tilde{N}_{\epsilon} u, \tilde{N}_{\epsilon} u, \tilde{M}_{\epsilon} u\right) \leq\left|\tilde{M}_{\epsilon} f\right|_{\epsilon}\left|\tilde{M}_{\epsilon} u\right|_{\epsilon} .
\end{array}
$$

Do lema 2.4, obtem-se

$$
\begin{array}{r}
b_{\epsilon}\left(\tilde{N}_{\epsilon} u, \tilde{N}_{\epsilon} u, \tilde{M}_{\epsilon} u\right) \leq \\
c_{4}|| \tilde{N}_{\epsilon} u||_{\epsilon}^{3 / 2}\left|A_{\epsilon} \tilde{N}_{\epsilon} u\right|_{\epsilon}^{1 / 2}\left|\tilde{M}_{\epsilon} u\right|_{\epsilon}
\end{array}
$$


onde a última igualdade acima vem de (28). Substituindo em (36),

$$
\begin{gathered}
\frac{1}{2} \frac{d}{d t}\left|\tilde{M}_{\epsilon} u\right|_{\epsilon}^{2}+\mu\left|A_{\epsilon}^{1 / 2} \tilde{M}_{\epsilon} u\right|_{\epsilon}^{2} \leq\left|\tilde{M}_{\epsilon} f\right|_{\epsilon}\left|\tilde{M}_{\epsilon} u\right|_{\epsilon}+ \\
c_{4}\left|A_{\epsilon}^{1 / 2} \tilde{N}_{\epsilon} u\right|_{\epsilon}^{3 / 2}\left|A_{\epsilon} \tilde{N}_{\epsilon} u\right|_{\epsilon}^{1 / 2}\left|\tilde{M}_{\epsilon} u\right|_{\epsilon} .
\end{gathered}
$$

A desigualdade de Poincarè, neste contexto, afirma que

$$
\left|\tilde{M}_{\epsilon} u\right|_{\epsilon} \leq \frac{1}{\lambda_{1}}\left|A_{\epsilon}^{1 / 2} \tilde{M}_{\epsilon} u\right|_{\epsilon}
$$

onde $\lambda_{1}$ é o primeiro autovalor (o menor) do operador de Stokes. Assim,

$$
\begin{array}{r}
\frac{d}{d t}\left|\tilde{M}_{\epsilon} u\right|_{\epsilon}^{2}+2 \mu\left|A_{\epsilon}^{1 / 2} \tilde{M}_{\epsilon} u\right|_{\epsilon}^{2} \leq \\
\frac{2}{\lambda_{1}}\left|\tilde{M}_{\epsilon} f\right|_{\epsilon}\left|A_{\epsilon}^{1 / 2} \tilde{M}_{\epsilon} u\right|_{\epsilon}+ \\
\frac{2 c_{4}}{\lambda_{1}}\left|A_{\epsilon}^{1 / 2} \tilde{N}_{\epsilon} u\right|_{\epsilon}^{3 / 2}\left|A_{\epsilon} \tilde{N}_{\epsilon} u\right|_{\epsilon}^{1 / 2}\left|A_{\epsilon}^{1 / 2} \tilde{M}_{\epsilon} u\right|_{\epsilon} .
\end{array}
$$

Para $\delta>0$,

$$
\begin{array}{r}
\frac{2}{\lambda_{1}}\left|\tilde{M}_{\epsilon} f\right|_{\epsilon}\left|A_{\epsilon}^{1 / 2} \tilde{M}_{\epsilon} u\right|_{\epsilon} \leq \\
2 \delta\left|A_{\epsilon}^{1 / 2} \tilde{M}_{\epsilon} u\right|_{\epsilon}^{2}+\frac{1}{2 \delta \lambda_{1}^{2}}\left|\tilde{M}_{\epsilon} f\right|_{\epsilon}^{2},
\end{array}
$$

e,

$$
\begin{array}{r}
\frac{2 c_{4}}{\lambda_{1}}\left|A_{\epsilon}^{1 / 2} \tilde{N}_{\epsilon} u\right|_{\epsilon}^{3 / 2}\left|A_{\epsilon} \tilde{N}_{\epsilon} u\right|_{\epsilon}^{1 / 2}\left|A_{\epsilon}^{1 / 2} \tilde{M}_{\epsilon} u\right|_{\epsilon} \leq \\
\frac{2}{4 \delta}\left(\frac{c_{4}}{\lambda_{1}}\left|A_{\epsilon}^{1 / 2} \tilde{N}_{\epsilon} u\right|_{\epsilon}^{3 / 2}\left|A_{\epsilon} \tilde{N}_{\epsilon} u\right|_{\epsilon}^{1 / 2}\right)^{2}+ \\
2 \delta\left|A_{\epsilon}^{1 / 2} \tilde{M}_{\epsilon} u\right|_{\epsilon}^{2}
\end{array}
$$

Logo,

$$
\begin{array}{r}
\frac{d}{d t}\left|\tilde{M}_{\epsilon} u\right|_{\epsilon}^{2}+2 \mu\left|A_{\epsilon}^{1 / 2} \tilde{M}_{\epsilon} u\right|_{\epsilon}^{2} \leq 2 \delta\left|A_{\epsilon}^{1 / 2} \tilde{M}_{\epsilon} u\right|_{\epsilon}^{2}+ \\
\frac{1}{2 \delta \lambda_{1}^{2}}\left|\tilde{M}_{\epsilon} f\right|_{\epsilon}^{2}+\frac{2}{4 \delta}\left(\frac{c_{4}}{\lambda_{1}}\left|A_{\epsilon}^{1 / 2} \tilde{N}_{\epsilon} u\right|_{\epsilon}^{3 / 2}\left|A_{\epsilon} \tilde{N}_{\epsilon} u\right|_{\epsilon}^{1 / 2}\right)^{2}+ \\
2 \delta\left|A_{\epsilon}^{1 / 2} \tilde{M}_{\epsilon} u\right|_{\epsilon}^{2}
\end{array}
$$

isto é,

$$
\begin{array}{r}
\frac{d}{d t}\left|\tilde{M}_{\epsilon} u\right|_{\epsilon}^{2}+(2 \mu-2 \delta-2 \delta)\left|A_{\epsilon}^{1 / 2} \tilde{M}_{\epsilon} u\right|_{\epsilon}^{2} \leq \\
\frac{1}{2 \delta \lambda_{1}^{2}}\left|\tilde{M}_{\epsilon} f\right|_{\epsilon}^{2}+\frac{c_{4}^{2}}{2 \delta \lambda_{1}^{2}}\left|A_{\epsilon}^{1 / 2} \tilde{N}_{\epsilon} u\right|_{\epsilon}^{3}\left|A_{\epsilon} \tilde{N}_{\epsilon} u\right|_{\epsilon}
\end{array}
$$

Seja $\delta$ de forma que $2 \mu-4 \delta=\mu$, ou seja, $\delta=\frac{\mu}{4}$. Daí,

$$
\frac{d}{d t}\left|\tilde{M}_{\epsilon} u\right|_{\epsilon}^{2}+\mu\left|A_{\epsilon}^{1 / 2} \tilde{M}_{\epsilon} u\right|_{\epsilon}^{2} \leq \frac{2}{\mu \lambda_{1}^{2}}\left|\tilde{M}_{\epsilon} f\right|_{\epsilon}^{2}+
$$

$$
\frac{2 c_{4}^{2}}{\mu \lambda_{1}^{2}}\left|A_{\epsilon}^{1 / 2} \tilde{N}_{\epsilon} u\right|_{\epsilon}^{3}\left|A_{\epsilon} \tilde{N}_{\epsilon} u\right|_{\epsilon}
$$

De (37),

$$
\begin{gathered}
\frac{d}{d t}\left|\tilde{M}_{\epsilon} u\right|_{\epsilon}^{2}+\mu \lambda_{1}\left|\tilde{M}_{\epsilon} u\right|_{\epsilon} \leq \frac{2}{\mu \lambda_{1}^{2}}\left|\tilde{M}_{\epsilon} f\right|_{\epsilon}^{2}+ \\
\frac{2 c_{4}^{2}}{\mu \lambda_{1}^{2}}\left|A_{\epsilon}^{1 / 2} \tilde{N}_{\epsilon} u\right|_{\epsilon}^{3}\left|A_{\epsilon} \tilde{N}_{\epsilon} u\right|_{\epsilon} .
\end{gathered}
$$

Aplicando a desigualdade de Gronwall, obtemos

$$
\begin{gathered}
\left|\tilde{M}_{\epsilon} u(t)\right|_{\epsilon}^{2} \leq a_{0}^{2}(\epsilon) e^{-\mu \lambda_{1} t}+\frac{\alpha^{2}(\epsilon)}{\mu^{2} \lambda_{1}^{3}}+ \\
\frac{2 c_{4}^{2}}{\mu \lambda_{1}^{2}} \int_{0}^{t}\left|A_{\epsilon}^{1 / 2} \tilde{N}_{\epsilon} u\right|_{\epsilon}^{3}\left|A_{\epsilon} \tilde{N}_{\epsilon} u\right|_{\epsilon} d s
\end{gathered}
$$

Do item (iii) do lema 3.1,

$$
\begin{array}{r}
\left|\tilde{M}_{\epsilon} u(t)\right|_{\epsilon}^{2} \leq a_{0}^{2}(\epsilon) e^{-\mu \lambda_{1} t}+\frac{\alpha^{2}(\epsilon)}{\mu^{2} \lambda_{1}^{3}}+ \\
\frac{2 c_{4}^{2}}{\mu \lambda_{1}^{2}} c_{5}(\mu) \epsilon\left(b_{0}^{2}(\epsilon)+\epsilon^{2} \beta^{2}(\epsilon)\right)\left(b_{0}^{2}(\epsilon)+t \beta^{2}(\epsilon)\right)
\end{array}
$$

Seja

$$
R_{n}^{2}(\epsilon)=\max \left(b_{0}^{2}(\epsilon), \beta^{2}(\epsilon)\right)
$$

Tem-se

$$
\begin{array}{r}
\epsilon\left(b_{0}^{2}(\epsilon)+\epsilon^{2} \beta^{2}(\epsilon)\right)\left(b_{0}^{2}(\epsilon)+t \beta^{2}(\epsilon)\right) \leq \\
\epsilon\left(2 \max \left(b_{0}^{2}(\epsilon), \beta^{2}(\epsilon)\right)\left(t \beta^{2}(\epsilon)\right)+b_{0}^{2}(\epsilon)\right) \leq \\
\epsilon 2 R_{n}^{2}(\epsilon)\left(t R_{n}^{2}(\epsilon)+R_{n}^{2}(\epsilon)\right) \\
\epsilon 2 R_{n}^{4}(\epsilon)(1+t) .
\end{array}
$$

Pondo $c_{6}(\mu)=\frac{4 c_{4}^{2}}{\mu \lambda_{1}^{2}} c_{5}(\mu)$ e substituindo em (41),

$$
\begin{aligned}
\left|\tilde{M}_{\epsilon} u(t)\right|_{\epsilon}^{2} & \leq a_{0}^{2}(\epsilon) e^{-\mu \lambda_{1} t}+\frac{\alpha^{2}(\epsilon)}{\mu^{2} \lambda_{1}^{3}} \\
& +c_{6}(\mu) \epsilon R_{n}^{4}(\epsilon)(1+t)
\end{aligned}
$$

o que prova o item (i) do lema.

Integrando de 0 à $t$ a expressão em (38),

$$
\begin{array}{r}
\left|\tilde{M}_{\epsilon} u(t)\right|_{\epsilon}^{2}+\mu \int_{0}^{t}\left|A_{\epsilon}^{1 / 2} \tilde{M}_{\epsilon} u(s)\right|_{\epsilon}^{2} d s \leq \\
\left|\tilde{M}_{\epsilon} u(0)\right|_{\epsilon}^{2}+\frac{2\left|\tilde{M}_{\epsilon} f\right|_{\epsilon}^{2} t}{\mu \lambda_{1}^{2}}+ \\
\frac{2 c_{4}^{2}}{\mu \lambda_{1}^{2}} \int_{0}^{t}\left|A_{\epsilon}^{1 / 2} \tilde{N}_{\epsilon} u\right|_{\epsilon}^{3}\left|A_{\epsilon} \tilde{N}_{\epsilon} u\right|_{\epsilon} d s .
\end{array}
$$

Segue que

$$
\mu \int_{0}^{t}\left|A_{\epsilon}^{1 / 2} \tilde{M}_{\epsilon} u(s)\right|_{\epsilon}^{2} d s \leq\left|\tilde{M}_{\epsilon} u(0)\right|_{\epsilon}^{2}+
$$




$$
\frac{2 \alpha^{2}(\epsilon) t}{\mu \lambda_{1}^{2}}+c_{6}(\mu) \epsilon R_{n}^{4}(\epsilon)(1+t)
$$

A partir de (37),

$$
\begin{aligned}
\int_{0}^{t}\left|A_{\epsilon}^{1 / 2} \tilde{M}_{\epsilon} u(s)\right|_{\epsilon}^{2} d s & \leq \frac{a_{0}^{2}(\epsilon)}{\mu \lambda_{1}}+\frac{2 \alpha^{2}(\epsilon) t}{\mu^{2} \lambda_{1}^{2}} \\
& +c_{6}(\mu) \epsilon R_{n}^{4}(\epsilon)(1+t),
\end{aligned}
$$

provando, assim, o item (ii) do lema.

Agora, para encontrar estimativas em $H^{1}$ para $\tilde{M}_{\epsilon}$, substituindo $v$ por $A_{\epsilon} u$ na formulação fraca de $\tilde{M}_{\epsilon}$, obtem-se

$$
\begin{array}{r}
\frac{d}{d t}\left(\tilde{M}_{\epsilon} u, \tilde{M}_{\epsilon} A_{\epsilon} u\right)_{\epsilon}+\mu\left(A_{\epsilon}^{1 / 2} \tilde{M}_{\epsilon} u, A_{\epsilon}^{1 / 2} \tilde{M}_{\epsilon} A_{\epsilon} u\right)_{\epsilon} \\
b_{\epsilon}\left(\tilde{M}_{\epsilon} u, \tilde{M}_{\epsilon} u, \tilde{M}_{\epsilon} A_{\epsilon} u\right)+b_{\epsilon}\left(\tilde{N}_{\epsilon} u, \tilde{N}_{\epsilon} u, \tilde{M}_{\epsilon} A_{\epsilon} u\right)= \\
\left(\tilde{M}_{\epsilon} f, \tilde{M}_{\epsilon} A_{\epsilon} u\right)_{\epsilon} .
\end{array}
$$

Procedendo analogamente aos cálculos iniciais feitos no lema 3.1, mas dessa vez para $\tilde{M}_{\epsilon}$, encontra-se as seguintes relações:

(i) $\frac{d}{d t}\left(\tilde{M}_{\epsilon} u, \tilde{M}_{\epsilon} A_{\epsilon} u\right)_{\epsilon}=\frac{1}{2} \frac{d}{d t}\left|A_{\epsilon}^{1 / 2} \tilde{M}_{\epsilon} u\right|_{\epsilon}^{2}$;

(ii) $\mu\left(A_{\epsilon}^{1 / 2} \tilde{M}_{\epsilon} u, A_{\epsilon}^{1 / 2} \tilde{M}_{\epsilon} A_{\epsilon} u\right)_{\epsilon}=\mu\left|A_{\epsilon} \tilde{M}_{\epsilon} u\right|_{\epsilon}^{2}$.

Logo,

$$
\begin{array}{r}
\frac{1}{2} \frac{d}{d t}\left|A_{\epsilon}^{1 / 2} \tilde{M}_{\epsilon} u\right|_{\epsilon}^{2}+\mu\left|A_{\epsilon} \tilde{M}_{\epsilon} u\right|_{\epsilon}^{2}= \\
\left(\tilde{M}_{\epsilon} f, \tilde{M}_{\epsilon} A_{\epsilon} u\right)_{\epsilon}-b_{\epsilon}\left(\tilde{M}_{\epsilon} u, \tilde{M}_{\epsilon} u, \tilde{M}_{\epsilon} A_{\epsilon} u\right) \\
b_{\epsilon}\left(\tilde{N}_{\epsilon} u, \tilde{N}_{\epsilon} u, \tilde{M}_{\epsilon} A_{\epsilon} u\right) .
\end{array}
$$

Usando o lema 2.2,

$$
\begin{array}{r}
\frac{d}{d t}\left|A_{\epsilon}^{1 / 2} \tilde{M}_{\epsilon} u\right|_{\epsilon}^{2}+2 \mu\left|A_{\epsilon} \tilde{M}_{\epsilon} u\right|_{\epsilon}^{2}= \\
2\left(\tilde{M}_{\epsilon} f, \tilde{M}_{\epsilon} A_{\epsilon} u\right)_{\epsilon}-2 b_{\epsilon}\left(\tilde{N}_{\epsilon} u, \tilde{N}_{\epsilon} u, \tilde{M}_{\epsilon} A_{\epsilon} u\right) .
\end{array}
$$

Aplicando o item (iii) do lema 2.4 à forma trilinear acima, temos

$$
\begin{aligned}
& 2 b_{\epsilon}\left(\tilde{N}_{\epsilon} u, \tilde{N}_{\epsilon} u, \tilde{M}_{\epsilon} A_{\epsilon} u\right) \leq \\
\leq & 2 c_{4}|| \tilde{N}_{\epsilon} u||_{\epsilon}^{3 / 2}\left|A_{\epsilon} \tilde{N}_{\epsilon} u\right|_{\epsilon}^{1 / 2}\left|A_{\epsilon} \tilde{M}_{\epsilon} u\right|_{\epsilon} \\
= & 2 c_{4}\left|A_{\epsilon}^{1 / 2} \tilde{N}_{\epsilon} u\right|_{\epsilon}^{3 / 2}\left|A_{\epsilon} \tilde{N}_{\epsilon} u\right|_{\epsilon}^{1 / 2}\left|A_{\epsilon} \tilde{M}_{\epsilon} u\right|_{\epsilon} \\
= & 2 c_{4} \frac{1}{\mathrm{k}}\left|A_{\epsilon}^{1 / 2} \tilde{N}_{\epsilon} u\right|_{\epsilon}^{3 / 2}\left|A_{\epsilon} \tilde{N}_{\epsilon} u\right|_{\epsilon}^{1 / 2} \mathrm{k}\left|A_{\epsilon} \tilde{M}_{\epsilon} u\right|_{\epsilon} \\
\leq & \left(\frac{1}{\mathrm{k}} c_{4}\left|A_{\epsilon}^{1 / 2} \tilde{N}_{\epsilon} u\right|_{\epsilon}^{3 / 2}\left|A_{\epsilon} \tilde{N}_{\epsilon} u\right|_{\epsilon}^{1 / 2}\right)^{2} \\
+ & \left(\mathrm{k}\left|A_{\epsilon} \tilde{M}_{\epsilon} u\right|_{\epsilon}\right)^{2},
\end{aligned}
$$

onde a última desigualdade acima foi obtida pela desigualdade de Young. A partir daí, seja $\mathrm{k}^{2}=\frac{\mu}{2}$, isto é, $\frac{1}{\mathrm{k}^{2}}=\frac{2}{\mu}$. Então,

$2 b_{\epsilon}\left(\tilde{N}_{\epsilon} u, \tilde{N}_{\epsilon} u, \tilde{M}_{\epsilon} A_{\epsilon} u\right) \leq \frac{2 c_{4}^{2}}{\mu}\left|A_{\epsilon}^{1 / 2} \tilde{N}_{\epsilon} u\right|_{\epsilon}^{3}\left|A_{\epsilon} \tilde{N}_{\epsilon} u\right|_{\epsilon}$

$$
+\frac{\mu}{2}\left|A_{\epsilon} \tilde{M}_{\epsilon} u\right|_{\epsilon}^{2}
$$

Ainda,

$$
\begin{array}{r}
2\left(\tilde{M}_{\epsilon} f, \tilde{M}_{\epsilon} A_{\epsilon} u\right)_{\epsilon} \leq 2\left|\tilde{M}_{\epsilon} f\right|_{\epsilon}\left|\tilde{M}_{\epsilon} A_{\epsilon} u\right|_{\epsilon}= \\
2 \mathrm{k}\left|\tilde{M}_{\epsilon} f\right|_{\epsilon} \frac{1}{\mathrm{k}}\left|\tilde{M}_{\epsilon} A_{\epsilon} u\right|_{\epsilon} \leq \\
\mathrm{k}^{2}\left|\tilde{M}_{\epsilon} f\right|_{\epsilon}^{2}+\frac{1}{\mathrm{k}^{2}}\left|\tilde{M}_{\epsilon} A_{\epsilon} u\right|_{\epsilon}^{2}= \\
\frac{2}{\mu}\left|\tilde{M}_{\epsilon} f\right|_{\epsilon}^{2}+\frac{\mu}{2}\left|\tilde{M}_{\epsilon} A_{\epsilon} u\right|_{\epsilon}^{2} .
\end{array}
$$

Novamente, usa-se aqui a desigualdade de Young e $\mathrm{k}^{2}=\frac{\mu}{2}$, isto é, $\frac{1}{\mathrm{k}^{2}}=\frac{2}{\mu}$. Substituindo (45) e (46) em (44),

$$
\begin{array}{r}
\frac{d}{d t}\left|A_{\epsilon}^{1 / 2} \tilde{M}_{\epsilon} u\right|_{\epsilon}^{2}+\mu\left|A_{\epsilon} \tilde{M}_{\epsilon} u\right|_{\epsilon}^{2} \leq \\
\frac{2}{\mu}\left|\tilde{M}_{\epsilon} f\right|_{\epsilon}^{2}+c_{7}\left|A_{\epsilon}^{1 / 2} \tilde{N}_{\epsilon} u\right|_{\epsilon}^{3}\left|A_{\epsilon} \tilde{N}_{\epsilon} u\right|_{\epsilon},
\end{array}
$$

onde $c_{7}(\mu)=\frac{2 c_{4}^{2}}{\mu}$.

Usando $A_{\epsilon}^{1 / 2} \tilde{M}_{\epsilon} u$ no lugar de $\tilde{M}_{\epsilon} u$ em (37), obtem-se

$$
\left|A_{\epsilon}^{1 / 2} \tilde{M}_{\epsilon} u\right|_{\epsilon} \leq \frac{1}{\lambda_{1}}\left|A_{\epsilon}^{1 / 2}\left(A_{\epsilon}^{1 / 2} \tilde{M}_{\epsilon} u\right)\right|_{\epsilon}
$$

ou seja,

$$
\left|A_{\epsilon}^{1 / 2} \tilde{M}_{\epsilon} u\right|_{\epsilon}^{2} \leq \frac{1}{\lambda_{1}^{2}}\left|A_{\epsilon} \tilde{M}_{\epsilon} u\right|_{\epsilon}^{2}
$$

Logo, de (47),

$$
\begin{array}{r}
\frac{d}{d t}\left|A_{\epsilon}^{1 / 2} \tilde{M}_{\epsilon} u\right|_{\epsilon}^{2}+\mu \lambda_{1}^{2}\left|A_{\epsilon}^{1 / 2} \tilde{M}_{\epsilon} u\right|_{\epsilon}^{2} \leq \\
\frac{2}{\mu}\left|\tilde{M}_{\epsilon} f\right|_{\epsilon}^{2}+c_{7}(\mu)\left|A_{\epsilon}^{1 / 2} \tilde{N}_{\epsilon} u\right|_{\epsilon}^{3}\left|A_{\epsilon} \tilde{N}_{\epsilon} u\right|_{\epsilon} .
\end{array}
$$

Com base na desigualdade de Gronwall, e procedendo analogamente aos cálculos que levam a desigualdade em (40), tem-se

$$
\begin{aligned}
\left|A_{\epsilon}^{1 / 2} \tilde{M}_{\epsilon} u\right|_{\epsilon}^{2} & \leq a_{0}^{2}(\epsilon) e^{-\mu \lambda_{1}^{2} t}+\frac{2 \alpha^{2}(\epsilon)}{\mu^{2} \lambda_{1}} \\
& +c_{8}(\mu) \epsilon R_{n}^{4}(\epsilon)(1+t)
\end{aligned}
$$

provando, assim, o item (iii) do lema. Integrando de 0 à $t$ a expressão em (47), prova-se o item (iv).

\subsection{Comportamento de $T^{\sigma}(\epsilon)$}

A partir de agora, demonstramos o resultado principal deste trabalho, isto é, o intervalo maximal de existência da solução forte das equações tridimensionais de Navier-Stokes, quando o domínio é considerado fino. Na seção anterior, foram demonstrados dois lemas com relação as estimativas para $\tilde{M}_{\epsilon}$ e $\tilde{N}_{\epsilon}$. O lema 3.1 vale 
para qualquer condição de fronteira a ser considerada. Entretanto, o lema 3.2 faz referência apenas para as condições (FF) e (FP). Esta distinção nas estimativas para $\tilde{M}_{\epsilon}$ decorre da definição da mesma; por exemplo, sabese que $\tilde{M}_{\epsilon} u=0$ para (DD) e (DP), isto é, não faz sentido calcular estimativas neste caso.

O primeiro resultado diz respeito as condições de fronteira (DD) e (DP).

Teorema 3.2. Suponha que

$$
\lim _{\epsilon \rightarrow 0} \epsilon\left(\left|A_{\epsilon}^{1 / 2} u_{0}\right|_{\epsilon}^{2}+|f|_{\epsilon}^{2}\right)=0 .
$$

Então, existe $\epsilon_{1}=\epsilon_{1}(\mu)$ tal que para $0<\epsilon<\epsilon_{1}$, a solução forte $u_{\epsilon}$ de

$$
\begin{cases}u_{t}+u \cdot \nabla u-\mu \Delta u+\nabla p=f, & \text { em } \Omega_{\epsilon} \times(0, \infty) \\ \operatorname{div} u=0, & \text { em } \Omega_{\epsilon} \times(0, \infty) \\ u(x, 0)=u_{0}(x), & \text { em } x \in \Omega_{\epsilon},\end{cases}
$$

com as condições de fronteira (DD) e (DP) existe para todo tempo, isto é, $T_{\epsilon}=\infty e$

$$
u_{\epsilon} \in C^{0}\left([0, \infty) ; V_{\epsilon}\right) \cap L^{2}\left(0, T, D\left(A_{\epsilon}\right)\right),
$$

para todo $T>0$.

Demonstração: Como definido inicialmente para as condições de fronteira (DD) e (DP), tem-se que $\tilde{M}_{\epsilon} u \equiv 0$. Deste modo, $\tilde{N}_{\epsilon} u=u$, onde $u \in L^{2}\left(\Omega_{\epsilon}\right)$ e, portanto, as estimativas encontradas no lema 3.1 valem também para $u$. Aplicando o item (i) do referido lema para a função $u$, obtem-se que

$$
\left|A_{\epsilon}^{1 / 2} u(t)\right|_{\epsilon}^{2} \leq\left|A_{\epsilon}^{1 / 2} u_{0}\right|_{\epsilon}^{2} e^{-\frac{\mu t}{2 \epsilon^{2}}}+\frac{2 \epsilon^{2}}{\mu^{2}}|f|_{\epsilon}^{2}
$$

Suponha que $T^{\sigma}(\epsilon)<\infty$. Para $\epsilon<\frac{\mu}{\sqrt{2}}$, vale que

$$
\left|A_{\epsilon}^{1 / 2} u_{0}\right|_{\epsilon}^{2} e^{-\frac{\mu t}{2 \epsilon^{2}}}+\frac{2 \epsilon^{2}}{\mu^{2}}|f|_{\epsilon}^{2} \leq\left|A_{\epsilon}^{1 / 2} u_{0}\right|_{\epsilon}^{2}+|f|_{\epsilon}^{2}
$$

pois $e^{-\frac{\mu t}{2 \epsilon^{2}}} \leq 1$. Daí,

$$
\left|A_{\epsilon}^{1 / 2} u(t)\right|_{\epsilon}^{2} \leq\left|A_{\epsilon}^{1 / 2} u_{0}\right|_{\epsilon}^{2}+|f|_{\epsilon}^{2} .
$$

Observe que, de (25) e (26),

$$
\sup _{0 \leq t \leq T^{\sigma}(\epsilon)}\left|A_{\epsilon}^{1 / 2} u(t)\right|_{\epsilon}^{2}=\sigma R_{0}^{2}(\epsilon) .
$$

Mas, por (49), $\left|A_{\epsilon}^{1 / 2} u_{0}\right|_{\epsilon}^{2}+|f|_{\epsilon}^{2}$ é uma cota superior para a função $\left|A_{\epsilon}^{1 / 2} u(t)\right|_{\epsilon}^{2}$. Logo,

$$
\sigma R_{0}^{2}(\epsilon) \leq\left|A_{\epsilon}^{1 / 2} u_{0}\right|_{\epsilon}^{2}+|f|_{\epsilon}^{2},
$$

para $\epsilon<\frac{\mu}{\sqrt{2}}$. Como, de (24)

$$
R_{0}^{2}(\epsilon)=\left|A_{\epsilon}^{1 / 2} u_{0}\right|_{\epsilon}^{2}+|f|_{\epsilon}^{2}
$$

a desigualdade acima implica que $\sigma \leq 1$, o que contradiz a suposição inicial de que $\sigma>1$. Portanto,

$$
T^{\sigma}(\epsilon)=+\infty,
$$

quando $\epsilon<\min \left(\frac{\mu}{\sqrt{2}}, \epsilon_{1}\right)$, onde $\epsilon_{1}$ satisfaz

$$
4 c_{4} \epsilon^{1 / 2} \sqrt{2 \sigma} R_{0}(\epsilon) \leq \frac{\mu}{2}
$$

para $0<\epsilon<\epsilon_{1}$.

Vale salientar que, para $t>0$ em (48), fazendo $\epsilon$ tender a zero, tem-se que

$$
\lim _{\epsilon \rightarrow 0}\left|A_{\epsilon}^{1 / 2} u_{t}\right|_{\epsilon}^{2} \leq \lim _{\epsilon \rightarrow 0}\left(\left|A_{\epsilon}^{1 / 2} u_{0}\right|_{\epsilon}^{2} e^{-\frac{\mu t}{2 \epsilon^{2}}}+\frac{2 \epsilon^{2}}{\mu^{2}}|f|_{\epsilon}^{2}\right)=0
$$

ou seja, $\forall t>0$,

$$
\lim _{\epsilon \rightarrow 0}\left|A_{\epsilon}^{1 / 2} u_{t}\right|_{\epsilon}^{2} \leq 0 \Rightarrow \lim _{\epsilon \rightarrow 0}\left|A_{\epsilon}^{1 / 2} u_{t}\right|_{\epsilon}^{2}=0
$$

a partir do qual se conclui que

$$
\lim _{\epsilon \rightarrow 0}\left\|u_{\epsilon}\right\|_{\epsilon}=0
$$

isto é, a norma em $H^{1}$ da solução $u_{\epsilon}$ converge para zero quando $\epsilon$ tende a zero, desde que $t>0$.

Com relação as condições (FF) e (FP), demonstra-se que $T^{\sigma}(\epsilon)=\infty$ em dois passos. Primeiro, tem-se o seguinte resultado:

Proposição 3.2. Considere as condições de fronteira $(F F) e$ (FP) e suponha que

$$
\lim _{\epsilon \rightarrow 0} \epsilon^{q}\left(\left|A_{\epsilon}^{1 / 2} u_{0}\right|_{\epsilon}^{2}+|f|_{\epsilon}^{2}\right)=0,
$$

para algum $0<q<1$. Então,

$$
\lim _{\epsilon \rightarrow 0} \epsilon^{2-2 q} T^{\sigma}(\epsilon)=\infty
$$

Demonstração: Dos lemas 3.1 e 3.2, tem-se

(i) $\left|A_{\epsilon}^{1 / 2} \tilde{N}_{\epsilon} u(t)\right|_{\epsilon}^{2} \leq b_{0}^{2}(\epsilon) e^{-\frac{\mu t}{2 \epsilon^{2}}}+\frac{2 \epsilon^{2}}{\mu^{2}} \beta^{2}(\epsilon)$ para $0 \leq t<T^{\sigma}(\epsilon)$ e

(ii) $\left|A_{\epsilon}^{1 / 2} \tilde{M}_{\epsilon} u(t)\right|_{\epsilon}^{2} \leq a_{0}^{2}(\epsilon) e^{-\mu \lambda_{1}^{2} t}+\frac{2 \alpha^{2}(\epsilon)}{\mu^{2} \lambda_{1}}$ $+c_{8}(\mu) \epsilon R_{n}^{4}(\epsilon)(1+t)$. 
Somando as duas expressões acima e usando o item (ii) do lema 2.1, temos

$$
\begin{aligned}
\left|A_{\epsilon}^{1 / 2} u(t)\right|_{\epsilon}^{2} & \leq b_{0}^{2}(\epsilon) e^{-\frac{\mu t}{2 \epsilon^{2}}}+\frac{2 \epsilon^{2}}{\mu^{2}} \beta^{2}(\epsilon)+a_{0}^{2}(\epsilon) e^{-\mu \lambda_{1}^{2} t} \\
& +\frac{\alpha^{2}(\epsilon)}{\mu^{2} \lambda_{1}}+c_{6}(\mu) \epsilon R_{n}^{4}(\epsilon)(1+t)
\end{aligned}
$$

Fixando $\sigma$ de modo que

$$
\sigma=4 \max \left(1, \frac{2}{\mu^{2}}, \frac{1}{\mu^{2} \lambda_{1}^{3}}\right)
$$

e, com isso, observe que

$$
\begin{aligned}
& b_{0}^{2}(\epsilon) \underbrace{e^{-\frac{\mu t}{2 \epsilon^{2}}}}_{<1}+\frac{2 \epsilon^{2}}{\mu^{2}} \beta^{2}(\epsilon)+a_{0}^{2}(\epsilon) \underbrace{e^{-\mu \lambda_{1} t}}_{<1}+\frac{\alpha^{2}(\epsilon)}{\mu^{2} \lambda_{1}^{3}} \leq \\
& b_{0}^{2} \frac{\sigma}{4}+\beta^{2}(\epsilon) \frac{\sigma}{4}+a_{0}^{2}(\epsilon) \frac{\sigma}{4}+\alpha^{2}(\epsilon) \frac{\sigma}{4}=R_{0}^{2}(\epsilon) \frac{\sigma}{4} .
\end{aligned}
$$

Substituindo em (51),

$$
\left|A_{\epsilon}^{1 / 2} u(t)\right|_{\epsilon}^{2} \leq R_{0}^{2}(\epsilon) \frac{\sigma}{4}+c_{6}(\mu) \epsilon R_{n}^{4}(\epsilon)(1+t) .
$$

Note ainda que, como $R_{n}^{2}(\epsilon)=\max \left(b_{0}^{2}(\epsilon), \beta^{2}(\epsilon)\right)$, temse

$$
R_{n}^{2}(\epsilon) \leq R_{0}^{2}(\epsilon) \Longrightarrow R_{n}^{4}(\epsilon) \leq R_{0}^{4}(\epsilon),
$$

a partir do qual

$$
\begin{gathered}
\left|A_{\epsilon}^{1 / 2} u(t)\right|_{\epsilon}^{2} \leq R_{0}^{2}(\epsilon) \frac{\sigma}{4}+c_{6}(\mu) \epsilon R_{n}^{4}(\epsilon)(1+t) \leq \\
R_{0}^{2}(\epsilon) \frac{\sigma}{4}+c_{6}(\mu) \epsilon R_{0}^{4}(\epsilon)(1+t)= \\
R_{0}^{2}(\epsilon) \frac{\sigma}{4}+c_{6}(\mu)\left(\epsilon^{q} R_{0}^{2}(\epsilon)\right) \epsilon^{1-q} R_{0}^{2}(\epsilon)(1+t),
\end{gathered}
$$

para $0 \leq t<T^{\sigma}(\epsilon)$.

Agora, suponha que $T^{\sigma}(\epsilon)<\infty$. Logo, de (26)

$$
\left|A_{\epsilon}^{1 / 2} u\left(T^{\sigma}(\epsilon)\right)\right|_{\epsilon}^{2}=\sigma R_{0}^{2}(\epsilon),
$$

e, substituindo em (53),

$$
\begin{aligned}
\frac{3 \sigma}{4} R_{0}^{2}(\epsilon) & \leq c_{6}(\mu)\left(\epsilon^{q} R_{0}^{2}(\epsilon)\right) \epsilon^{1-q} R_{0}^{2}(\epsilon)(1 \\
& \left.+T^{\sigma}(\epsilon)\right) .
\end{aligned}
$$

Não é difícil ver que $R_{0}^{2}(\epsilon) \neq 0$. De fato, se esta não fosse válida, isto é, se $R_{0}^{2}(\epsilon)=0$, de (24)

$$
\left|A_{\epsilon}^{1 / 2} u_{0}\right|_{\epsilon}^{2}=0 \text { e }|f|_{\epsilon}^{2}=0
$$

Assim, $f=0$ e $A_{\epsilon}^{1 / 2} u_{0}=0$ q.s. em $\Omega_{\epsilon}$. Daí $u_{0}=0$ q.s. o que implicaria $u(t)=0$, uma solução trivial a qual não interessa. Logo, $R_{0}^{2}(\epsilon) \neq 0$ e, portanto, de (54),

$$
\frac{3 \sigma}{4} \leq c_{6}(\mu)\left(\epsilon^{q} R_{0}^{2}(\epsilon)\right) \epsilon^{1-q}\left(1+T^{\sigma}(\epsilon)\right) .
$$

Afirma-se que

$$
\lim _{\epsilon \rightarrow 0} \epsilon^{1-q} T^{\sigma}(\epsilon)=\infty
$$

Suponha que a expressão acima não seja verdade, ou seja, existe uma constante $L_{1}>0$ tal que para todo $\delta>0$, tem-se $\epsilon>0$ com $0<\epsilon<\delta$ e $\epsilon^{1-q} T^{\sigma}(\epsilon) \leq L_{1}$.

Sendo assim, tome $\delta=\frac{1}{n}, n \geq 1$. Então, existe uma sequência $\left(\epsilon_{n}\right)_{n \geq 1}$ com

$$
\lim _{n \rightarrow \infty} \epsilon_{n}=0,
$$

pois $0<\epsilon<\frac{1}{n}$ e $\epsilon_{n}^{1-q} T^{\sigma}\left(\epsilon_{n}\right) \leq L_{1}$, isto é,

$$
T^{\sigma}\left(\epsilon_{n}\right) \leq \frac{L_{1}}{\epsilon_{n}^{1-q}}, \forall n \geq 1 .
$$

Voltando na expressão (55),

$$
\frac{3 \sigma}{4} \leq c_{6}(\mu)\left(\epsilon_{n}^{q} R_{0}^{2}\left(\epsilon_{n}\right)\right) \epsilon_{n}^{1-q}\left(1+\frac{L_{1}}{\epsilon_{n}^{1-q}}\right) .
$$

Mas $0<q<1$ e $\epsilon_{n}<1$, daí, $1-q>0$ e $\epsilon_{n}^{1-q}<1$. Logo,

$$
\begin{aligned}
\frac{3 \sigma}{4} & \leq c_{6}(\mu)\left(\epsilon_{n}^{q} R_{0}^{2}\left(\epsilon_{n}\right)\right)\left(1+L_{1}\right) \\
\frac{3 \sigma}{4} & \leq L_{2} \epsilon_{n}^{q} R_{0}^{2}\left(\epsilon_{n}\right)
\end{aligned}
$$

onde $L_{2}=L_{2}(\mu)=c_{6}(\mu)\left(1+L_{1}\right)$. Fazendo $n$ tender ao infinito e usando a hipótese (50), o lado direito da expressão (57) tende a zero e, portanto, $\sigma=0$, uma contradição com a escolha de $\sigma$ em (52). Logo, vale (56) e a proposição está demonstrada.

Agora, mostra-se que o intervalo maximal de existência da solução forte das equações de Navier-Stokes é infinito, quando o domínio é fino. Mais precisamente,

Teorema 3.3. Suponha que

$$
\lim _{\epsilon \rightarrow 0} \epsilon^{2 q}\left(\left|A_{\epsilon}^{1 / 2} u_{0}\right|_{\epsilon}^{2}+|f|_{\epsilon}^{2}\right)=0
$$

para algum $0<2 q<1$. Então, existe $\epsilon_{2}=\epsilon_{2}(\mu, q, \omega)$ tal que para $0<\epsilon<\epsilon_{2}$, a solução forte $u_{\epsilon}$ de

$$
\begin{cases}u_{t}+u \cdot \nabla u-\mu \Delta u+\nabla p=f, & \text { em } \Omega_{\epsilon} \times(0, \infty) \\ \operatorname{div} u=0, & \text { em } \Omega_{\epsilon} \times(0, \infty) \\ u(x, 0)=u_{0}(x), & \text { em } x \in \Omega_{\epsilon},\end{cases}
$$

com as condições de fronteira (FF) e (FP) existe para todo tempo, isto é, $T_{\epsilon}=\infty e$

$$
u_{\epsilon} \in C^{0}\left([0, \infty) ; V_{\epsilon}\right) \cap L^{2}\left(0, T, D\left(A_{\epsilon}\right)\right),
$$

para todo $T>0$. 


\section{Demonstração: Seja}

$$
K_{\epsilon}^{2}=\left|A_{\epsilon}^{1 / 2} \tilde{M}_{\epsilon} u_{0}\right|_{\epsilon}^{2}+\frac{8}{\mu^{2} \lambda_{1}}\left|\tilde{M}_{\epsilon} f\right|_{\epsilon}^{2}+B_{\epsilon}^{2}
$$

onde

$$
B_{\epsilon}^{2}=\left|A_{\epsilon}^{1 / 2} \tilde{N}_{\epsilon} u_{0}\right|_{\epsilon}^{2}+\left|\tilde{N}_{\epsilon} f\right|_{\epsilon}^{2} .
$$

Observe que, substituindo a expressão acima em $K_{\epsilon}^{2}$, aplicando o item (ii) do lema 2.1 e a hipótese (58) temos

$$
\begin{aligned}
\lim _{\epsilon \rightarrow 0} \epsilon^{q} K_{\epsilon}^{2} & =\lim _{\epsilon \rightarrow 0} \epsilon^{q}\left(\left|A_{\epsilon}^{1 / 2} u_{0}\right|_{\epsilon}^{2}+|f|_{\epsilon}^{2}\right) \\
& =0
\end{aligned}
$$

Agora, escolhe-se $\epsilon_{2}=\epsilon_{2}\left(\mu, \lambda_{1}, q\right)$ satisfazendo

(i) $0<\epsilon_{2}<\frac{1}{4}$;

(ii) Para $0<\epsilon \leq \epsilon_{2}, \epsilon^{q} K_{\epsilon}^{2} \leq 1$;

(iii) $\frac{2 \epsilon^{2}}{\mu^{2}} \leq \frac{1}{8}, e^{-\frac{\mu \lambda_{1}}{\epsilon^{2(1-q)}}} \leq \frac{1}{4} \mathrm{e}$ $\frac{16 c_{4}(\mu)}{\mu} \max \left(1, \frac{1}{\mu^{3}}\right) \epsilon^{q} \leq \frac{1}{4} ;$

(iv) Para $0<\epsilon \leq \epsilon_{2}, \epsilon^{2-2 q} T^{\sigma}(\epsilon)>4$.

Note que o item (ii) decorre de (59) e, no item (iii), o lado esquerdo das desigualdades tendem a zero a medida que $\epsilon$ se aproxima de zero. Pela proposição 3.2, o lado esquerdo da expressão em (iv) tende ao infinito, tornando válido tal item. Sendo assim, está bem definido este $\epsilon_{2}$.

Lembrando o item (iii) do lema 3.1, pode-se reescrever tal estimativa com os dados acima, isto é,

$$
\begin{aligned}
\int_{0}^{t}\left|A_{\epsilon}^{1 / 2} \tilde{N}_{\epsilon} u(s)\right|_{\epsilon}^{3}\left|A_{\epsilon} \tilde{N}_{\epsilon} u(s)\right|_{\epsilon} d s & \leq \\
c_{5}(\mu) \epsilon\left(b_{0}^{2}(\epsilon)+\epsilon^{2} \beta^{2}(\epsilon)\right)\left(b_{0}^{2}(\epsilon)+t \beta^{2}(\epsilon)\right) & \leq \\
c_{5}(\mu) \epsilon\left(\epsilon^{2} B_{\epsilon}^{2}+B_{\epsilon}^{2}\right)\left(t B_{\epsilon}^{2}+B_{\epsilon}^{2}\right) & = \\
c_{5}(\mu) \epsilon B_{\epsilon}^{4}\left(\epsilon^{2}+1\right)(1+t) & \leq \\
\frac{4}{\mu} \max \left(1, \frac{1}{\mu^{3}}\right) B_{\epsilon}^{4}(1+t) . &
\end{aligned}
$$

Defina, para $0<\epsilon \leq \epsilon_{2}$,

$$
t_{\epsilon}=\epsilon^{2(q-1)} .
$$

Do lema 3.1, item (i),

$$
\left|A_{\epsilon}^{1 / 2} \tilde{N}_{\epsilon} u(t)\right|_{\epsilon}^{2} \leq b_{0}^{2}(\epsilon) e^{-\frac{\mu t}{2 \epsilon^{2}}}+\frac{2 \epsilon^{2}}{\mu^{2}} \beta^{2}(\epsilon),
$$

e como $e^{-\frac{\mu t}{2 \epsilon^{2}}} \leq e^{-\frac{\mu \epsilon_{\epsilon}}{2 \epsilon^{2}}}$, onde $t_{\epsilon} \leq t<2 t_{\epsilon}$, vale que

$$
\left|A_{\epsilon}^{1 / 2} \tilde{N}_{\epsilon} u(t)\right|_{\epsilon}^{2} \leq b_{0}^{2}(\epsilon) e^{-\frac{\mu t_{\epsilon}}{2 \epsilon^{2}}}+\frac{2 \epsilon^{2}}{\mu^{2}} \beta^{2}(\epsilon),
$$

onde $t_{\epsilon} \leq t<2 t_{\epsilon}$. Assim, usando as condições satisfeitas por $\epsilon_{2}$,

$$
\begin{aligned}
\left|A_{\epsilon}^{1 / 2} \tilde{N}_{\epsilon} u(t)\right|_{\epsilon}^{2} & \leq b_{0}^{2} \frac{1}{4}+\beta^{2}(\epsilon) \frac{1}{8} \\
& =b_{0}^{2}\left(\frac{1}{8}+\frac{1}{8}\right)+\beta^{2}(\epsilon) \frac{1}{8} \\
& \leq B_{\epsilon}^{2} \frac{1}{8}+B_{\epsilon}^{2} \frac{1}{8} \\
& =\frac{1}{4} B_{\epsilon}^{2}
\end{aligned}
$$

ou seja,

$$
\left|A_{\epsilon}^{1 / 2} \tilde{N}_{\epsilon} u(t)\right|_{\epsilon}^{2} \leq \frac{1}{4} B_{\epsilon}^{2},
$$

para $t_{\epsilon} \leq t<2 t_{\epsilon}$.

Agora, usando o item (iii) do lema 3.2, isto é,

$$
\begin{aligned}
\left|A_{\epsilon}^{1 / 2} \tilde{M}_{\epsilon} u(t)\right|_{\epsilon}^{2} & \leq a_{0}^{2}(\epsilon) e^{-\mu \lambda_{1}^{2} t}+\frac{2 \alpha^{2}(\epsilon)}{\mu^{2} \lambda_{1}} \\
& +c_{8}(\mu) \epsilon R_{n}^{4}(\epsilon)(1+t)
\end{aligned}
$$

e recordando que $R_{n}^{2}(\epsilon)=\max \left(b_{0}^{2}(\epsilon), \beta^{2}(\epsilon)\right)$, obtem-se

$$
\begin{aligned}
& \left|A_{\epsilon}^{1 / 2} \tilde{M}_{\epsilon} u(t)\right|_{\epsilon}^{2} \leq a_{0}^{2}(\epsilon) e^{-\mu \lambda_{1}^{2} t_{\epsilon}}+\frac{2 \alpha^{2}(\epsilon)}{\mu^{2} \lambda_{1}} \\
+ & \mathrm{c}(\mu) \epsilon\left(\epsilon^{q} B_{\epsilon}^{2}\right) B_{\epsilon}^{2} \epsilon^{1-q}\left(1+2 t_{\epsilon}\right),
\end{aligned}
$$

para $t_{\epsilon} \leq t<2 t_{\epsilon}$, onde $\mathrm{c}(\mu)=\frac{16 c_{4}(\mu)}{\mu} \max \left(1, \frac{1}{\mu^{3}}\right)$. Usando as condições satisfeitas por $\epsilon_{2}$, tem-se

$$
\begin{aligned}
a_{0}^{2}(\epsilon) e^{-\mu \lambda_{1}^{2} t_{\epsilon}} & \leq \frac{1}{4} K_{\epsilon}^{2} \quad \text { e } \\
\frac{2 \alpha^{2}(\epsilon)}{\mu^{2} \lambda_{1}} \leq \frac{1}{8} K_{\epsilon}^{2} & \leq \frac{1}{4} K_{\epsilon}^{2} .
\end{aligned}
$$

Além disso, observe que $\epsilon^{q} B_{\epsilon}^{2} \leq \epsilon^{q} K_{\epsilon}^{2} \leq 1$ e portanto,

$$
\mathrm{c}(\mu) \epsilon\left(\epsilon^{q} B_{\epsilon}^{2}\right) B_{\epsilon}^{2} \epsilon^{1-q}\left(1+2 t_{\epsilon}\right) \leq \frac{3}{4} B_{\epsilon}^{2} .
$$

Substituindo (65) e (64) em (63),

$$
\left|A_{\epsilon}^{1 / 2} \tilde{M}_{\epsilon} u(t)\right|_{\epsilon}^{2} \leq \frac{5}{4} K_{\epsilon}^{2}
$$

para $t_{\epsilon} \leq t<2 t_{\epsilon}$. Somando as expressões (66) e (61), novamente aplicando o item (ii) do lema 2.1,

$$
\left|A_{\epsilon}^{1 / 2} u(t)\right|_{\epsilon}^{2} \leq \frac{3}{2} K_{\epsilon}^{2}
$$

Sabe-se que a norma de uma aplicação é uma função contínua. Isto vale também para a norma $\|\cdot\|_{\epsilon}$. Posto isso, calculando o limite quando $t$ tende à $t_{\epsilon}$, para a função $\left|A_{\epsilon}^{1 / 2} u(t)\right|_{\epsilon}^{2}$, tem-se

$$
\lim _{t \rightarrow t_{\epsilon}}\left|A_{\epsilon}^{1 / 2} u(t)\right|_{\epsilon}^{2}=\lim _{t \rightarrow t_{\epsilon}}\|u(t)\|_{\epsilon}^{2}
$$




$$
\begin{aligned}
& =\left\|u\left(t_{\epsilon}\right)\right\|_{\epsilon}^{2} \\
& =\left|A_{\epsilon}^{1 / 2} u\left(2 t_{\epsilon}\right)\right|_{\epsilon}^{2}
\end{aligned}
$$

e como

$$
\lim _{t \rightarrow t_{\epsilon}}\left|A_{\epsilon}^{1 / 2} u(t)\right|_{\epsilon}^{2} \leq \frac{3}{2} K_{\epsilon}^{2}
$$

segue que

$$
\left|A_{\epsilon}^{1 / 2} u\left(2 t_{\epsilon}\right)\right|_{\epsilon}^{2} \leq \frac{3}{2} K_{\epsilon}^{2}
$$

O objetivo agora é usar indução. Suponha que existe um tempo $t_{0}$ satisfazendo

$$
\begin{gathered}
\left|A_{\epsilon}^{1 / 2} \tilde{N}_{\epsilon} u\left(t_{0}\right)\right|_{\epsilon}^{2} \leq \frac{1}{2} B_{\epsilon}^{2} \quad \mathrm{e} \\
\left|A_{\epsilon}^{1 / 2} \tilde{M}_{\epsilon} u\left(t_{0}\right)\right|_{\epsilon}^{2} \leq \frac{1}{2} K_{\epsilon}^{2} .
\end{gathered}
$$

A desigualdade de Gronwall para o intervalo $t_{0} \leq t<$ $T^{\sigma}(\epsilon)$ faz com que as expressões (60) e (62) sofram alguma mudança. Sendo assim, temos

$$
\begin{aligned}
\left|A_{\epsilon}^{1 / 2} \tilde{N}_{\epsilon} u(t)\right|_{\epsilon}^{2} & \leq e^{-\frac{\mu\left(t-t_{0}\right)}{2 \epsilon^{2}}}\left|A_{\epsilon}^{1 / 2} \tilde{N}_{\epsilon} u\left(t_{0}\right)\right|_{\epsilon}^{2} \\
& +\frac{2 \epsilon^{2}}{\mu^{2}} \beta^{2}(\epsilon),
\end{aligned}
$$

e, por (67),

$$
\left|A_{\epsilon}^{1 / 2} \tilde{N}_{\epsilon} u(t)\right|_{\epsilon}^{2} \leq \frac{1}{2} B_{\epsilon}^{2} e^{-\frac{\mu\left(t-t_{0}\right)}{2 \epsilon^{2}}}+\frac{2 \epsilon^{2}}{\mu^{2}} \beta^{2}(\epsilon)
$$

$\operatorname{com} t_{0} \leq t<T^{\sigma}(\epsilon)$.

Também

$$
\begin{gathered}
\left|A_{\epsilon}^{1 / 2} \tilde{M}_{\epsilon} u(t)\right|_{\epsilon}^{2} \leq e^{-\mu \lambda_{1}^{2}\left(t-t_{0}\right)}\left|A_{\epsilon}^{1 / 2} \tilde{M}_{\epsilon} u\left(t_{0}\right)\right|_{\epsilon}^{2} \\
+\frac{2 \alpha^{2}(\epsilon)}{\mu^{2} \lambda_{1}}+c_{8}(\mu) \epsilon R_{n}^{4}(\epsilon)\left(1+t-t_{0}\right) .
\end{gathered}
$$

De (67),

$$
\begin{aligned}
\left|A_{\epsilon}^{1 / 2} \tilde{M}_{\epsilon} u(t)\right|_{\epsilon}^{2} & \leq \frac{1}{2} K_{\epsilon}^{2} e^{-\mu \lambda_{1}^{2}\left(t-t_{0}\right)}+\frac{2 \alpha^{2}(\epsilon)}{\mu^{2} \lambda_{1}} \\
& +c_{8}(\mu)\left(\epsilon^{q} B_{\epsilon}^{2}\right) B_{\epsilon}^{2} \epsilon^{1-q}\left(1+t-t_{0}\right)
\end{aligned}
$$

$\operatorname{com} t_{0} \leq t<T^{\sigma}(\epsilon)$.

Tome, portanto, $t_{0}=2 \epsilon^{2(q-1)}$. Assim como feito em (66) e (61), obtêm-se

$$
\begin{aligned}
\left|A_{\epsilon}^{1 / 2} \tilde{N}_{\epsilon} u(t)\right|_{\epsilon}^{2} & \leq \frac{1}{8} B_{\epsilon}^{2}+\frac{1}{8} B_{\epsilon}^{2} \\
& =\frac{1}{4} B_{\epsilon}^{2}
\end{aligned}
$$

e

$$
\left|A_{\epsilon}^{1 / 2} \tilde{M}_{\epsilon} u(t)\right|_{\epsilon}^{2} \leq \frac{1}{8} K_{\epsilon}^{2}+\frac{1}{8} K_{\epsilon}^{2}+\frac{1}{4} K_{\epsilon}^{2}
$$

$$
\begin{aligned}
& =\frac{1}{2} K_{\epsilon}^{2} \\
\operatorname{para} 2 \epsilon^{2(q-1)} \leq t & <3 \epsilon^{2(q-1)} \cdot \text { Logo, } \\
\left|A_{\epsilon}^{2} u(t)\right|_{\epsilon}^{2} & =\left|A_{\epsilon}^{1 / 2} \tilde{N}_{\epsilon} u(t)\right|_{\epsilon}^{2}+\left|A_{\epsilon}^{1 / 2} \tilde{M}_{\epsilon} u(t)\right|_{\epsilon}^{2} \\
& \leq \frac{1}{4} B_{\epsilon}^{2}+\frac{1}{2} K_{\epsilon}^{2} \\
& \leq \frac{1}{4} K_{\epsilon}^{2}+\frac{1}{2} K_{\epsilon}^{2} \\
& =\frac{3}{4} K_{\epsilon}^{2}
\end{aligned}
$$

para $2 \epsilon^{2(q-1)} \leq t<3 \epsilon^{2(q-1)}$, o que implica $T^{\sigma}(\epsilon)>$ $3 \epsilon^{2(q-1)}$.

Analogamente, para $t_{0}=3 \epsilon^{2(q-1)}$, tem-se

$$
\left|A_{\epsilon}^{2} u(t)\right|_{\epsilon}^{2} \leq \frac{3}{4} K_{\epsilon}^{2}
$$

para $3 \epsilon^{2(q-1)} \leq t<4 \epsilon^{2(q-1)}$, a partir do qual $T^{\sigma}(\epsilon)>$ $4 \epsilon^{2(q-1)}$. Repetindo este argumento $n$ vezes, concluí-se que

$$
T^{\sigma}(\epsilon)>n \epsilon^{2(q-1)}, \quad \forall n \geq 1 .
$$

Portanto, $T^{\sigma}(\epsilon)=+\infty$ para $0<\epsilon \leq \epsilon_{2}$, ou seja, o intervalo maximal de existência da solução forte para o sistema de equações de Navier-Stokes em domínios tridimenisonais fino é infinito.

\section{Conclusões}

O estudo sobre as equações de Navier-Stokes é importante para a matemática não somente pelo seu amplo campo de aplicações, mas para melhor entendimento e desenvolvimento de teorias no campo das equações diferenciais parciais. Este trabalho teve como objetivo compilar resultados que levaram ao estudo do intervalo maximal de existência da solução das equações de Navier-Stokes em domínios tridimensionais finos do tipo $\Omega_{\epsilon}=\omega \times(0, \epsilon)$, com $\omega \subset \mathbb{R}^{2}$ e $\epsilon \in(0,1)$.

$\mathrm{O}$ principal resultado obtido neste artigo depende do teorema clássico de existência de soluções para domínios limitados quaisquer, o qual garante, com certas hipóteses, a existência de solução fraca. Restringindo os dados iniciais, tal teorema garante também a existência de uma solução única, denominada solução forte, para um tempo $T>0$ que depende do domínio, dos dados iniciais e da viscosidade. Com isso, podemos concluir que em domínios finos, dependendo de uma relação entre os dados iniciais, este tempo $T$ é infinito, ou seja, o intervalo maximal de existência de solução forte para as equações de Navier-Stokes é infinito. 


\section{Referências}

Adams, R. A., Fournier, J. J. (2003). Sobolev spaces, vol 140. Academic press.

Brezis, H. (1983). Analyse fonctionelle, vol 5. Masson.

Chorin, A. J., Marsden, J. E. (1990). A mathematical introduction to fluid mechanics, vol 2. Springer New York.

Evans, L. (2010). Partial Differential Equations. Graduate studies in mathematics, American Mathematical Society.

Foias, C., Manley, I., Rosa, R., Temam, R., Mayer, M. E. (2002). Navier-Stokes equations and turbulence, vol 55. Taylor \& Francis.

Fox, R., McDonald, A. (1988). Introdução a mecânica dos fluidos. $3^{\text {a }}$. Ed Rio de Janeiro: Ed Guanabara.

Kreiss, H. O., Lorenz, J. (1989). Initial-boundary value problems and the Navier-Stokes equations, vol 136. Access Online via Elsevier.

Lions, J. L. (1969). Quelques méthodes de résolution des problemes aux limites non linéaires, vol 31. Dunod Paris.

Melo, S. T., Neto, F. M. (1991). Mecânica dos fluidos e equaçoes diferenciais. IMPA.

Raugel, G., Sell, G. R. (1989). Equations de navier-stokes dans des domaines minces en dimension trois: régularité globale. Comptes rendus de l'Académie des sciences Série 1, Mathématique, 309(6), 299-303.

Rudin, W. (1986). Real and complex analysis (3rd). New York: McGraw-Hill Inc.

Temam, R. (1984). Navier-Stokes Equations. American Mathematical Soc..

Temam, R. (1995). Navier-Stokes equations and nonlinear functional analysis, vol 66. Siam.

Temam, R., Ziane, M. (1996). Navier-stokes equations in three-dimensional thin domains with various boundary conditions. Advances in Differential Equations, 1(4), 499-546. 